\title{
STAR FORMATION NEAR BERKELEY 59: EMBEDDED PROTOSTARS
}

\author{
J. M. Rosvick ${ }^{1}$ and D. Majaess ${ }^{2}$ \\ ${ }^{1}$ Department of Physical Sciences, Thompson Rivers University, 900 McGill Road, Kamloops, BC V2C 0C8, Canada \\ ${ }^{2}$ Department of Astronomy and Physics, Saint Mary's University, Halifax, NS B3H 3C3, Canada \\ Received 2013 April 30; accepted 2013 September 16; published 2013 October 25
}

\begin{abstract}
A group of suspected protostars in a dark cloud northwest of the young ( $\sim 2 \mathrm{Myr})$ cluster Berkeley 59 and two sources in a pillar south of the cluster have been studied in order to determine their evolutionary stages and ascertain whether their formation was triggered by Berkeley 59. Narrowband near-infrared observations from the Observatoire du Mont Mégantic, ${ }^{12} \mathrm{CO}(J=3-2)$ and SCUBA-2 (450 and $\left.850 \mu \mathrm{m}\right)$ observations from the JCMT, 2MASS, and WISE images, and data extracted from the IPHAS survey catalog were used. Of 12 sources studied, two are Class I objects, while three others are flat/Class II, one of which is a T Tauri candidate. A weak CO outflow and two potential starless cores are present in the cloud, while the pillar possesses substructure at different velocities, with no outflows present. The CO spectra of both regions show peaks in the range $v_{\mathrm{LSR}}=-15$ to $-17 \mathrm{~km} \mathrm{~s}^{-1}$, which agrees with the velocity adopted for Berkeley $59\left(-15.7 \mathrm{~km} \mathrm{~s}^{-1}\right)$, while spectral energy distribution models yield an average interstellar extinction $A_{V}$ and distance of $15 \pm 2$ mag and $830 \pm 120 \mathrm{pc}$, respectively, for the cloud, and $6.9 \mathrm{mag}$ and $912 \mathrm{pc}$ for the pillar, indicating that the regions are in the same vicinity as Berkeley 59. The formation of the pillar source appears to have been triggered by Berkeley 59. It is unclear whether Berkeley 59 triggered the association's formation.
\end{abstract}

Key words: infrared: stars - stars: protostars - submillimeter: stars

Online-only material: color figures

\section{INTRODUCTION}

$\mathrm{H}$ II regions and stellar winds from $\mathrm{OB}$ associations have the potential to trigger star formation by applying sustained pressure to surrounding material (Elmegreen 2009). The structures that form from this material (which may be remnants of the molecular cloud that produced the OB association) take on the appearance of pillars and shells. Often, the pillars possess bright rims that point toward the energy source, and there may be at least one young stellar object (YSO) embedded in the structure. The shells that delineate the edge of the $\mathrm{H}$ II ionization front may contain embedded objects, as was observed by Zavagno et al. (2006), for example, in their study of the H II region RCW 79; they investigated four condensations of material at millimeter and near-infrared wavelengths and discovered several embedded massive YSOs, the formation of which appears to have been triggered by the collect-and-collapse process first proposed by Elmegreen \& Lada's (1977) study of OB associations, in which winds and ionizing radiation from the first generation of massive stars disperse the remnants of the parent cloud, and once the density of this material reaches a critical value, a new generation of stars is formed. Conversely, Koenig et al. (2012), using Wide-Field Infrared Survey Explorer (WISE; Wright et al. 2010) mid-IR data, analyzed several star-forming regions possessing pillars and clouds of varying masses surrounding a central excavated cavity and found that the spatial and class distribution of YSOs was more indicative of the radiatively driven implosion (RDI) process outlined by Reipurth (1983) in a study of cometary clouds in the Gum Nebula. In this scenario, the winds and radiation from the first generation of stars disperse the remnants of the cloud, only to reveal higher-density regions (e.g., pillars) that erode more slowly than the surroundings. The subsequent pressure on these regions condenses the material, which leads to the next generation of star formation. See Elmegreen (2011) for a thorough review of the literature regarding the triggering processes.
Indicators of protostellar age/evolutionary status for both low- and high-mass protostars include the presence of carbon monoxide (CO) outflows and $\mathrm{Br} \gamma$ and $\mathrm{H} \alpha$ emission. Curtis et al. (2010) detected outflows toward several protostellar cores and found that the morphology and velocity of the outflows correlated with protostellar evolutionary stage. $\mathrm{Br} \gamma$ emission is an indicator of the accretion of circumstellar material in classical T Tauri stars (CTTSs; Najita et al. 1996), while the strength of the $\mathrm{Br} \gamma$ emission line correlates with the mass accretion rate (Muzerolle et al. 1998). Recently, Barentsen et al. (2011) conducted an Isaac Newton Telescope Photometric $\mathrm{H} \alpha$ (IPHAS; Drew et al. 2005) survey of IC 1396 and found several CTTS candidates that exhibited $\mathrm{H} \alpha$ emission of varying strengths.

Cep OB4 is a large star-forming complex that contains several molecular clouds, the H II region Sharpless 171, other emission regions, and the young ( $\sim 2 \mathrm{Myr})$ star cluster Berkeley 59 (Figure 1, leftmost image). That cluster hosts an O5V star (BD $\left.+66^{\circ} 1673\right)$ and several member stars earlier than spectral type B8 (Kun et al. 2008, and references therein). Cep OB4 has been studied extensively, and areas of both lowand intermediate-mass star formation have been discovered. MacConnell (1968) studied $\mathrm{H} \alpha$ emission stars in the nebulosity surrounding Berkeley 59 and concluded that many of them are potential T Tauri stars, while Yang et al. (1990) surveyed the complex in ${ }^{13} \mathrm{CO}(J=1-0)$ and discovered a molecular cloud containing outflows toward two IRAS sources, the IRAS luminosities of which indicated that they are low-mass YSOs associated with the cloud. Pandey et al. (2008) used Two Micron All-Sky Survey (2MASS) point-source catalog (Skrutskie et al. 2006) data and slitless spectroscopy to study several T Tauri stars in the vicinity of Berkeley 59, while Yang \& Fukui (1992) mapped an extensive $\left(l=115^{\circ}-125^{\circ}, b=0^{\circ}-10^{\circ}\right)$ region in the $J=1-0$ transition of ${ }^{12} \mathrm{CO},{ }^{13} \mathrm{CO}$, and $\mathrm{C}^{18} \mathrm{O}$ surrounding Berkeley 59 and discovered two high-density clumps that have been compressed laterally by the radiation pressure from 

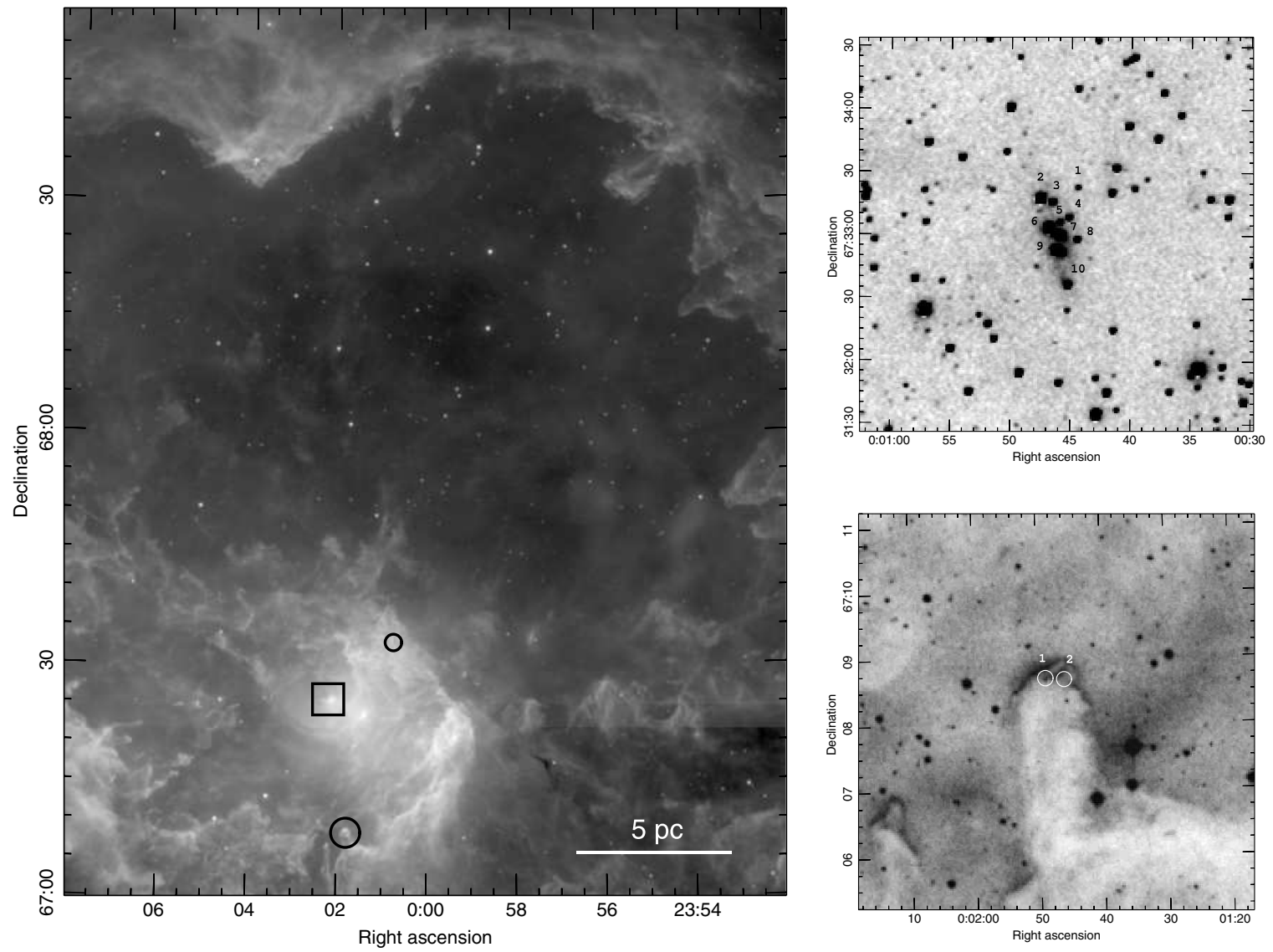

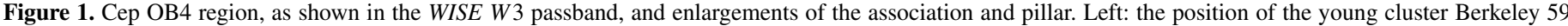

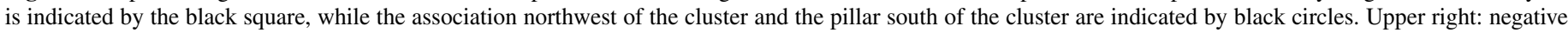

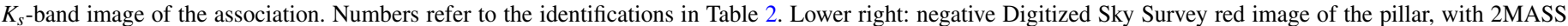
detections indicated by open circles. Numbers refer to the identifications in Table 2.

massive cluster stars. Pandey et al. (2008) claimed their results as evidence for second-generation star formation that may have been triggered by the massive stars in the cluster, while Yang \& Fukui (1992) suggested that second-generation star formation may occur in the compressed clumps within the next Myr or so, provided that the clumps do not dissipate within that time (we have shown in this work that star formation actually has occurred already in one of the clumps). The conclusions of these investigations were supported by Koenig et al.'s (2012) extensive study of star-forming regions mentioned above, which included the Berkeley 59 region (however, they did not focus on the objects studied here). Those authors found the YSOs to be rather uniformly distributed about the cluster (in particular the O-type stars; see their Figure 1(c)), and an analysis of the distribution suggests that the YSO formation was triggered by the cluster, as opposed to forming passively.

Despite extensive mapping of the region around Berkeley 59, two areas have been overlooked for further, detailed investigation, and which may be used in part to support one of the triggering mechanisms. While visually inspecting $\mathrm{Ob}$ servatoire du Mont Mégantic (OMM) $K_{s}$-band images of Berkeley 59 that were obtained for a different study, we noticed a small association of objects lying some 12 arcmin northwest of the cluster and a pillar located south of it. The association, at $00^{\mathrm{h}} 00^{\mathrm{m}} 46^{\mathrm{s}},+67^{\circ} 32^{\prime} 59^{\prime \prime}(\mathrm{J} 2000.0)$, resides in a cloud within the clump named C2 studied by Yang \& Fukui (1992). This group is not apparent on optical images. The $2 \mathrm{MASS}$ point-source cata$\log$ lists 10 sources in the association, contained within an area
$1 \operatorname{arcmin}^{2}$, and following an elongated arrangement (Figure 1, upper right). YSOs in embedded clusters may follow elongated spatial distributions that often parallel structures in the parent molecular cloud (Gutermuth et al. 2009). The cloud in which the association resides is elongated roughly north-south, with an eastern ridge that appears to have been formed by pressure from the massive stars of Berkeley 59 and a western ridge that may have been formed by pressure from a first generation of massive stars (now dispersed) that also created the cavity observed to the north of Berkeley 59 (Figure 1, leftmost image). Several of these objects are protostellar in nature: all of them possess infrared (IR) excesses, three were detected in all four WISE passbands (one was detected in three of the passbands), and two were detected by AKARI (previously known as IRIS, the Infrared Imaging Surveyor; Ishihara et al. 2010). The clump in which the association resides appears to be physically associated with Berkeley 59 on the basis of velocity studies: Yang \& Fukui (1992) found that the local standard-of-rest velocity $\left(v_{\mathrm{LSR}}\right)$ of the clump $\left(-16 \mathrm{~km} \mathrm{~s}^{-1}\right)$ matches the velocity of Berkeley 59 $\left(-15.7 \mathrm{~km} \mathrm{~s}^{-1}\right)$ derived from observations of member stars (Liu et al. 1989).

The cloud in which the association resides was searched for other YSOs, and despite the detection of several red sources in both the 2MASS and WISE catalogs, the mid-IR colors suggested that nearly all of these are star-forming galaxies ([3.4]-[4.6] $\sim 0.5 \mathrm{mag}$, [4.6]-[12] between 3 and 5 mag; see Section 3.1 for an explanation of this analysis technique). Only a few additional potential YSOs were found, but since they were 
located in another part of the cloud, they were excluded from this study.

The pillar, some 18 arcmin south of Berkeley 59 at $00^{\mathrm{h}} 01^{\mathrm{m}} 46^{\mathrm{s}}$, $+67^{\circ} 08^{\prime} 40^{\prime \prime}$ (J2000.0), exhibits a bright rim on its northernmost edge and non-uniform structure (Figure 1, lower right). There are several 2MASS and WISE sources present. While most of these are reddened background sources possessing mid-IR colors indicative of either background stars or star-forming galaxies, two sources near the rim exhibit IR excesses whose mid-IR colors are suggestive of YSOs; their positions are indicated by white circles. Extensive ${ }^{12} \mathrm{CO}(J=1-0)$ mapping by Leisawitz et al. (1989) indicated that the velocity range is widespread over a large region surrounding Berkeley 59, but since the antenna resolution of their telescope was 8.7 arcmin and the pillar is less than 2 arcmin across in right ascension, the pillar was not resolved. Incidentally, the pillar was not resolved in Yang \& Fukui's (1992) maps either; the beam size of that study was 2.7 arcmin. Note that a smaller pillar resides a few arcmin east of this one. Gahm et al. (2006) mapped it in $\mathrm{H} \alpha$, ${ }^{12} \mathrm{CO}$ and ${ }^{13} \mathrm{CO}$ (both $J=1-0$ ), and $\mathrm{HCO}^{+}$and found its velocity to be $v_{\mathrm{LSR}}=-16.4 \mathrm{~km} \mathrm{~s}^{-1}$, again in agreement with that of Berkeley 59. It is assumed that the larger pillar's velocity is comparable in value, considering the proximity of both pillars to the cluster.

It appears as if these overlooked regions, which probably are associated with Berkeley 59 on the basis of the velocities, contain protostars but it is not clear which stage of evolution they currently are in, and whether their formation was triggered by the cluster stars. A definitive result would add further insight to the wealth of knowledge regarding triggering mechanisms. To that end, we obtained for both regions narrowband $\mathrm{Br} \gamma$ and continuum observations using the OMM via queue mode observing (Artigau et al. 2010) and HARP (Buckle et al. 2009) ${ }^{12} \mathrm{CO}(J=3-2)$ submillimeter observations using the James Clerk Maxwell telescope (JCMT; flexible queue observing). The association region was also observed with the SCUBA-2 (Holland et al. 2013) continuum detector on the JCMT (flexible queue observing). The data from WISE and the Mid-course Space Experiment (MSX; Egan et al. 2003) were used to fill in the wavelength range between the near-IR and submillimeter data, for the purpose of fitting spectral energy distributions (SEDs). Galactic plane surveys such as that conducted by the Spitzer Space Telescope did not reach high enough in Galactic latitude to include the association and pillar, and while the IRAS catalog contained some detections in the point-source rejection catalog, and there was extended emission across much of the Berkeley 59 region, there were no point-source detections in either the association region or the pillar. Section 2 describes the observations, while the analysis is presented in Section 3. The results are discussed in Section 4 and summarized in Section 5.

\section{OBSERVATIONS AND DATA REDUCTION}

\subsection{Archived Data}

The 2MASS and WISE image servers ${ }^{3}$ were used to download Atlas images (sizes of 136 and $8640 \mathrm{arcmin}^{2}$ for 2MASS and WISE, respectively) containing the association and pillar. Source extraction and profile-fitting photometry were performed with DAOPHOT (Stetson 1987). Calibrations to the natural photometric system of 2MASS were determined as follows. For each passband, astrometric positions and catalog identifications were obtained for each detection. Uncrowded stars were

\footnotetext{
http://irsa.ipac.caltech.edu/
}

Table 1

Derived 2MASS Zero Points

\begin{tabular}{lcc}
\hline \hline Passband & Association & Pillar \\
\hline$J$ & $-4.12 \pm 0.03$ & $-4.01 \pm 0.05$ \\
$H$ & $-4.64 \pm 0.05$ & $-4.61 \pm 0.06$ \\
$K_{S}$ & $-4.96 \pm 0.05$ & $-4.91 \pm 0.05$ \\
\hline
\end{tabular}

cross-matched with the corresponding catalog objects, and the resulting lists were cleaned to remove blends, saturated stars, poor-quality detections, and artifacts. Zero points were calculated by taking the means of the magnitude differences in the sense [standard - instrumental] and applied to the instrumental photometry. Table 1 lists the zero points for the 2MASS passbands. The WISE instrumental magnitudes were calibrated using the pipeline's zero points $(20.5,19.5,18.0$, and 13.0 for $W 1, W 2, W 3$, and $W 4$, respectively), since the instrument was very stable, and the zero points were calculated using thousands of sources. We were unable to generate zero points of the same quality using only the fields downloaded (especially for $W 3$ and $W 4$, where there were very few calibrating sources available).

Table 2 lists the sources and their 2MASS $J H K_{s}$ magnitudes (narrowband OMM results, discussed in Section 2.2, are included as well). A letter/number ID system ( $\mathrm{A}=$ association, $\mathrm{P}=$ pillar) is introduced to avoid confusion when the objects are discussed in the text. For completeness, magnitudes corresponding to undetected objects are given as 2MASS upper limits (indicated by colons following the magnitudes). Objects A2, A3, and A6 had 2MASS photometric quality flags of A or $\mathrm{B}$ (signal-to-noise ratio of 10 or 7 , respectively) for all three passbands. The others, with the exception of A9, had quality flags of A or B for the $K_{s}$ band only. This could be an indication of protostellar evolutionary stage, given that earlier-class objects are more deeply embedded in their parent clouds and thus are detectible only at longer wavelengths. Note that while A9 was reduced as a single object in the 2MASS data reduction pipeline, it actually is a blend of two objects that was apparent in all of the near-IR images. This has been indicated in Table 2 by the letters $a$ and $b$. Quoted uncertainties are those obtained by adding in quadrature the uncertainties from profile-fitting and of the zero points given in Table 1 .

Table 3 presents the results of the mid-IR profile-fitting photometry. Magnitude uncertainties were obtained by adding in quadrature the profile-fitting and zero-point uncertainties given in the WISE documentation. Submillimeter (SCUBA-2, discussed in Section 2.4) data for both regions are given as well. A2 and A7 also appeared in the AKARI-IRC Point Source Catalogue (Kataza et al. 2010). P1 was present in the MSX and AKARI-IRC Point Source Catalogues, while P2 did not appear in any mid-IR catalogs. Only sources for which there are both nearand mid-IR data are considered in the subsequent analysis. The $A K A R I$ data were not used since the passbands were not included in Robitaille et al.'s (2007) SED fitter tool.

\subsection{Narrowband Infrared Data}

Continuum $\left(\lambda_{0}=2.255\right.$ and $2.033 \mu \mathrm{m}$, having widths of $\Delta \lambda=0.10$ and $0.025 \mu \mathrm{m}$, hereafter referred to as CONT1 and CONT2, respectively) and $\operatorname{Br} \gamma\left(\lambda_{0}=2.165 \mu \mathrm{m}, \Delta \lambda=\right.$ $0.02 \mu \mathrm{m})$ images were obtained on 2011 September 11 and November 8 (UT) via queue mode observing with the CPAPIR detector mounted on the $1.6 \mathrm{~m}$ Ritchey-Chrétien telescope at the OMM. This detector contains a $2048 \times 2048$ array of 0.89 arcsec pixels, with a field of view of $30 \mathrm{arcmin}^{2}$. Standard IR observing 
Table 2

Near-infrared 2MASS and OMM Narrowband Data

\begin{tabular}{|c|c|c|c|c|c|c|c|}
\hline Figure ID & 2MASS ID & $J$ & $H$ & $K_{s}$ & $m_{\mathrm{Br} \gamma}$ & $m_{\mathrm{CONT} 1}$ & $m_{\mathrm{CONT} 2}$ \\
\hline A1 & $00004441+6733225$ & 17.66: & $16.30 \pm 0.38$ & $15.04 \pm 0.21$ & $15.15 \pm 0.04$ & $15.08 \pm 0.06$ & $15.25 \pm 0.06$ \\
\hline A2 & $00004754+6733171$ & $15.18 \pm 0.06$ & $12.78 \pm 0.06$ & $11.31 \pm 0.06$ & $11.47 \pm 0.03$ & $11.36 \pm 0.04$ & $11.82 \pm 0.03$ \\
\hline A3 & $00004651+6733159$ & $16.20 \pm 0.11$ & $14.49 \pm 0.09$ & $13.50 \pm 0.07$ & $13.62 \pm 0.03$ & $13.45 \pm 0.03$ & $13.61 \pm 0.03$ \\
\hline A4 & $00004514+6733085$ & 17.37: & $15.40 \pm 0.17$ & $13.94 \pm 0.09$ & $14.03 \pm 0.03$ & $13.80 \pm 0.04$ & $14.35 \pm 0.03$ \\
\hline A5 & $00004588+6733063$ & $16.98 \pm 0.22$ & $15.95 \pm 0.30$ & $14.26 \pm 0.13$ & $14.64 \pm 0.04$ & $14.42 \pm 0.04$ & $15.13 \pm 0.05$ \\
\hline A6 & $00004685+6733035$ & $15.97 \pm 0.11$ & $13.40 \pm 0.07$ & $11.81 \pm 0.06$ & $11.44 \pm 0.03$ & $11.37 \pm 0.05$ & $11.78 \pm 0.03$ \\
\hline A7 & $00004584+6733000$ & $17.53 \pm 0.39$ & $15.78 \pm 0.28$ & $13.63 \pm 0.10$ & $13.80 \pm 0.06$ & $13.53 \pm 0.03$ & $14.20 \pm 0.11$ \\
\hline A8 & $00004449+6732583$ & 17.51: & $16.25 \pm 0.33$ & $14.12 \pm 0.11$ & $14.08 \pm 0.03$ & $13.95 \pm 0.03$ & $14.60 \pm 0.04$ \\
\hline A9a & $00004629+6732525$ & $15.52 \pm 0.08$ & $13.53 \pm 0.07$ & $12.22 \pm 0.06$ & $12.22 \pm 0.04$ & $12.14 \pm 0.05$ & $12.57 \pm 0.03$ \\
\hline A9b & $00004629+6732525$ & $16.19 \pm 0.14$ & $13.87 \pm 0.08$ & $12.53 \pm 0.06$ & $12.50 \pm 0.05$ & $12.47 \pm 0.04$ & $12.97 \pm 0.05$ \\
\hline A10 & $00004530+6732363$ & 17.62: & $15.17 \pm 0.16$ & $12.88 \pm 0.07$ & $13.40 \pm 0.04$ & $13.14 \pm 0.05$ & $13.99 \pm 0.05$ \\
\hline P1 & $00014876+6708405$ & $13.13 \pm 0.05$ & $11.39 \pm 0.06$ & $9.98 \pm 0.06$ & $9.47 \pm 0.03$ & $10.81 \pm 0.25$ & $9.69 \pm 0.01$ \\
\hline $\mathrm{P} 2$ & $00014637+6708396$ & $16.84 \pm 0.21$ & $15.41 \pm 0.20$ & $14.00 \pm 0.12$ & $14.37 \pm 0.06$ & $14.36 \pm 0.11$ & $14.37 \pm 0.09$ \\
\hline
\end{tabular}

Note. Magnitudes followed by colons refer to 2MASS upper limit magnitudes.

Table 3

Mid-infrared and Submillimeter Data

\begin{tabular}{|c|c|c|c|c|c|c|c|c|}
\hline \multirow{2}{*}{$\begin{array}{l}\text { Figure } \\
\text { ID }\end{array}$} & \multicolumn{4}{|c|}{ WISE } & \multicolumn{2}{|c|}{$M S X$} & \multicolumn{2}{|c|}{ SCUBA-2 } \\
\hline & $\begin{array}{c}W 1(3.4 \mu \mathrm{m}) \\
(\mathrm{mag})\end{array}$ & $\begin{array}{c}W 2(4.6 \mu \mathrm{m}) \\
(\mathrm{mag})\end{array}$ & $\begin{array}{c}W 3(12 \mu \mathrm{m}) \\
(\mathrm{mag})\end{array}$ & $\begin{array}{c}W 4(22 \mu \mathrm{m}) \\
(\mathrm{mag})\end{array}$ & $\begin{array}{c}A(8.3 \mu \mathrm{m}) \\
(\mathrm{Jy})\end{array}$ & $\begin{array}{c}D(14.7 \mu \mathrm{m}) \\
(\mathrm{Jy})\end{array}$ & $\begin{array}{l}S_{450} \\
(\mathrm{Jy})\end{array}$ & $\begin{array}{l}S_{850} \\
(\mathrm{Jy})\end{array}$ \\
\hline $\mathrm{A} 2$ & $9.65 \pm 0.03$ & $8.66 \pm 0.04$ & $6.19 \pm 0.05$ & $4.08 \pm 0.06$ & $\ldots$ & $\ldots$ & $\ldots$ & $\ldots$ \\
\hline A6 & $10.07 \pm 0.03$ & $9.28 \pm 0.04$ & $7.27 \pm 0.05$ & 4.74: & $\cdots$ & $\cdots$ & $\cdots$ & $\cdots$ \\
\hline A7 & $11.05 \pm 0.04$ & $9.03 \pm 0.04$ & $6.25 \pm 0.05$ & $2.48 \pm 0.06$ & $\ldots$ & $\ldots$ & $1.19 \pm 0.07$ & $0.121 \pm 0.008$ \\
\hline A10 & $11.33 \pm 0.03$ & $9.42 \pm 0.04$ & $6.88 \pm 0.05$ & $4.99 \pm 0.07$ & $\ldots$ & $\ldots$ & $0.55 \pm 0.07$ & $0.075 \pm 0.008$ \\
\hline $\mathrm{P} 1$ & $8.16 \pm 0.03$ & $7.22 \pm 0.04$ & $4.98 \pm 0.05$ & $1.98 \pm 0.06$ & $0.63 \pm 0.03$ & $0.89 \pm 0.09$ & $\ldots$ & $\cdots$ \\
\hline
\end{tabular}

procedures were followed, with several $40 \mathrm{~s}$ dithered images taken, reduced, and co-added using a data reduction pipeline developed by the Laboratory of Experimental Astrophysics team at the Université de Montréal. This procedure combines the frames using a mix of median and mean, with the output image behaving much like a straight mean in terms of noise characteristics (É. Artigau 2011, private communication). Mean stellar FWHM values were 1.72, 2.00, and 1.76 arcsec, and midexposure air masses were 1.12, 1.13, and 1.09, for $\mathrm{Br} \gamma, \mathrm{CONT} 1$, and CONT2, respectively.

The source extraction, photometry, and matching to the corresponding 2MASS $K_{s}$ detections were conducted in a similar fashion to the archived data, with least-squares regression performed in order to obtain transformation equations (this approach yielded better results than the straight zero-point determination method employed for the other IR data). Table 4 lists the coefficients and their uncertainties, along with the goodnessof-fit parameter $R$. Equations are in the form of $Y=a X+b$. Final calibrated photometry is given in Table 2. Quoted uncertainties are from profile fitting; standard errors obtained from the least-squares analysis range from 0.09 to $0.11 \mathrm{mag}$.

\subsection{Submillimeter Data: HARP}

${ }^{12} \mathrm{CO}(J=3-2)$ data were obtained via the CANSERV observing program ${ }^{4}$ on 2011 August 11 UT using the HARP receiver and ACSIS backend on the JCMT, in order to verify the presence of any outflows. HARP consists of 16 receptors that are separated by 30 arcsec; at the time of the observations the H12 receptor was not operational. A single sub-band centered at $345.8 \mathrm{GHz}$, with bandwidth $250 \mathrm{MHz}$ and channel spacing

\footnotetext{
4 CANSERV is a service observing program that is intended for short or urgent projects that do not initially require a full observing proposal.
}

$30.5 \mathrm{kHz}$ (corresponding to $0.026 \mathrm{~km} \mathrm{~s}^{-1}$ ), was used to obtain the observations. The HARP4 jiggle pattern with default spacing of $7.5 \mathrm{arcsec}$ was used to produce maps 2 arcmin on a side.

The maps were centered on $00^{\mathrm{h}} 00^{\mathrm{m}} 47^{\mathrm{s}},+67^{\circ} 33^{\prime} 04^{\prime \prime}$ and $00^{\mathrm{h}} 01^{\mathrm{m}} 48^{\mathrm{s}},+67^{\circ} 08^{\prime} 41^{\prime \prime}(\mathrm{J} 2000.0)$ for the association and pillar, respectively. Since there were no emission-free regions close to the sources, the position-switch mode was employed, with the off position located at $23^{\mathrm{h}} 58^{\mathrm{m}} 50^{\mathrm{s}},+68^{\circ} 42^{\prime} 00^{\prime \prime}$, determined by examining the ${ }^{12} \mathrm{CO} J=1-0$ maps constructed by Leisawitz et al. (1989). This off position was used for both regions.

The zenith opacity at $225 \mathrm{GHz}$ averaged 0.1 (weather band 3), and total integration time was 18 minutes per region; the sources themselves were observed for just over 11 minutes each. Data were obtained and are presented on the antenna temperature scale $T_{\mathrm{A}}^{*}$. The estimated receiver temperature $T_{\mathrm{rx}}$ was $90 \mathrm{~K}$, and median system temperatures $T_{\text {sys }}$ were $452 \mathrm{~K}$ and $466 \mathrm{~K}$ for the association and pillar maps, respectively.

The raw time series data were processed at the Canadian Astronomy Data Center with the ORAC Data Reduction (ORAC-DR) pipeline (Cavanagh et al. 2008), using the SMURF COMmand MAKECUBE (with the ORAC-DR recipe REDUCE SCIENCE_GRADIENT) in the Starlink software package (Chapin et al. 2013). The resulting maps were rebinned to a velocity resolution of $0.26 \mathrm{~km} \mathrm{~s}^{-1}$ and smoothed with a Gaussian of $\mathrm{FWHM}=3$ pixels, which yielded a final rms noise of $\sigma_{T_{\mathrm{A}}^{*}}$ ranging from 0.038 to $0.061 \mathrm{~K}$ for the association and 0.059 to $0.087 \mathrm{~K}$ for the pillar.

\subsection{Submillimeter Data: SCUBA-2}

Simultaneous 450 and $850 \mu \mathrm{m}$ continuum observations of the association were obtained with SCUBA-2 on 2012 August 16, 19, and 21 (UT) using the Daisy mapping pattern. The scan velocity along the scan axis was $155 \operatorname{arcsec~s}^{-1}$, with scan 
Table 4

Results of a Least-squares Analysis of the Instrumental 2MASS and OMM Narrowband Photometry

\begin{tabular}{|c|c|c|c|c|c|c|}
\hline \multirow[t]{2}{*}{ Passband } & \multicolumn{3}{|c|}{ Association } & \multicolumn{3}{|c|}{ Pillar } \\
\hline & $a$ & $b$ & $R$ & $a$ & $b$ & $R$ \\
\hline $\mathrm{Br} \gamma$ & $1.019 \pm 0.003$ & $-0.26 \pm 0.04$ & 0.995 & $1.007 \pm 0.003$ & $-0.06 \pm 0.03$ & 0.998 \\
\hline CONT1 & $1.018 \pm 0.004$ & $-0.11 \pm 0.06$ & 0.989 & $1.061 \pm 0.004$ & $-0.71 \pm 0.05$ & 0.995 \\
\hline CONT2 & $1.009 \pm 0.002$ & $-0.11 \pm 0.03$ & 0.996 & $0.988 \pm 0.002$ & $-0.22 \pm 0.03$ & 0.997 \\
\hline
\end{tabular}

spacing perpendicular to the scan axis of 0.6 arcsec. The total area mapped was roughly 16 arcmin in diameter; final maps were cropped as discussed below.

Six observations of the region (each consisting of 65 subscans of $35 \mathrm{~s}$ duration) were obtained, for a total of close to $4 \mathrm{hr}$ of observing time (which includes object as well as dark and flatfield scans). Currently accepted values of the half-power beam width are 7.9 and 13.0 arcsec for the main beam, and 25.0 and 48.0 arcsec for the error beam, at 450 and $850 \mu \mathrm{m}$, respectively (Dempsey et al. 2013). Zenith opacities at $225 \mathrm{GHz}$ ranged from 0.034 to 0.074 .

The data were processed using the SMURF Dynamic Iterative Map-Maker (DIMM) in the Starlink data reduction package. This routine pre-cleans the data and iteratively reduces the cleaned data to produce a final map. The configuration file CONFIG_BRIGHT_EXTENDED was used since bright sources as well as extended emission were present in the images. The DIMM down-sampled the 450 and $850 \mu \mathrm{m}$ data to 2 and 4 pixels, respectively, to preserve information in the resulting maps. In an attempt to lessen the negative bowling (dark areas surrounding the strongest sources) present in the data, external masks were constructed and applied to the data according to the method described in the SCUBA-2 reduction manual. ${ }^{5}$

Initially the 450 and $850 \mu \mathrm{m}$ maps were calibrated to the traditional $\mathrm{Jy} \mathrm{beam}^{-1}$ units using flux conversion factors (FCFs) of 491 and $537 \mathrm{Jy} \mathrm{pW}^{-1}$ beam $^{-1}$, respectively (Dempsey et al. 2013), and cropped to the central 3 arcmin. Figure 2 displays the cropped $450 \mu \mathrm{m}$ map (in the negative). Peak emission at the center of the map is at a value of $900 \mathrm{mJy}^{\mathrm{beam}}{ }^{-1}$. Bowls in the vicinity of the emission (described earlier, and appearing as brighter regions in Figure 2) are at a value of $-100 \mathrm{mJy}^{\text {beam }}{ }^{-1}$. The rms level in the map is $22.2 \mathrm{mJy}$. Association objects are indicated by the open circles, and CO contours (discussed in Section 3.4) are superposed. Since flux densities for the extended sources initially believed to be associated with objects A7 and A10 were required to anchor the long-wavelength end of the SED, the maps were also calibrated using FCFs appropriate for aperture photometry. Currently accepted FCF values (for a 60 arcsec diameter aperture) of 4.71 and $2.34 \mathrm{Jy} \mathrm{pW}^{-1} \operatorname{arcsec}^{-2}$ for 450 and $850 \mu \mathrm{m}$, respectively, were scaled to a target aperture of 24 arcsec diameter using the curve of growth provided in the SCUBA-2 reduction manual. The rms levels in these maps (cropped to the central 3 arcmin) were 0.170 and $0.007 \mathrm{mJy}$, respectively. This procedure was followed since the sources were extended, the aperture size encompassed the sources fully, and the contribution from the error beam at $450 \mu \mathrm{m}$ was taken into account automatically (the $850 \mu \mathrm{m}$ error beam flux outside the aperture was not taken into account since its peak level was less than $2 \%$ of the main beam and the correction would have been insignificant).

The photometry was performed using the same aperture ( 24 arcsec diameter) for both maps, for both A7 and A10, by

\footnotetext{
5 http://www.starlink.ac.uk/docs/sc21.htx/sc21.html
}

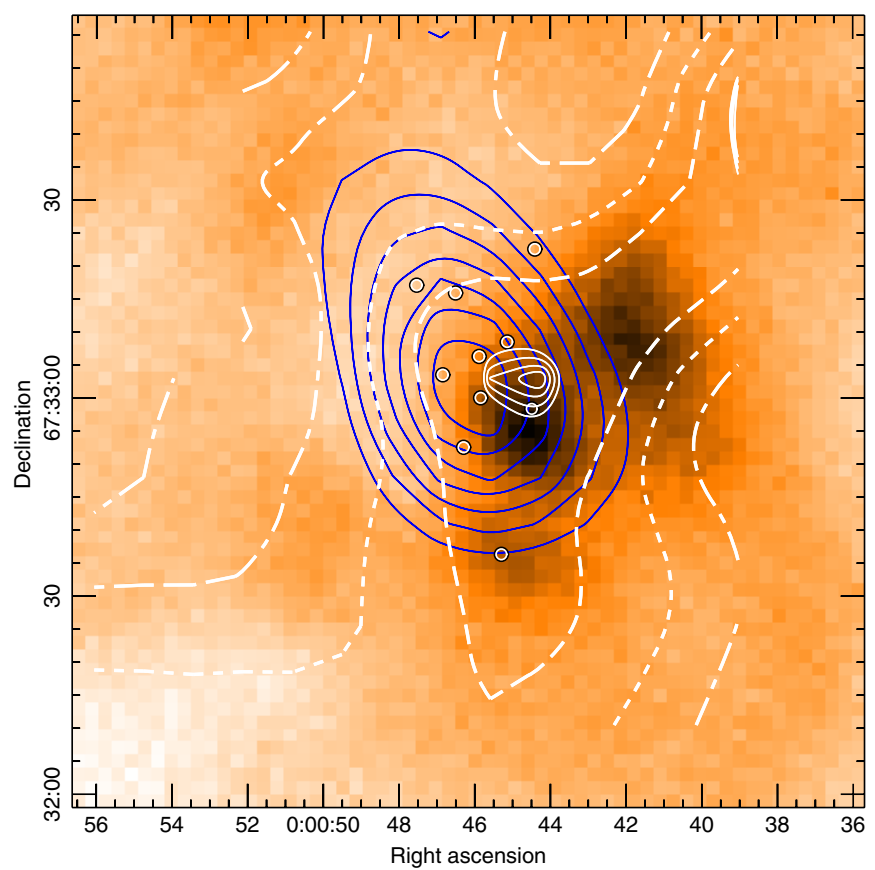

Figure 2. Cropped SCUBA- $2450 \mu \mathrm{m}$ map, shown in the negative. The SCUBA2 intensities run from -100 to $900 \mathrm{mJy}^{\text {beam }}{ }^{-1}$. Association objects are indicated by the open circles. Solid dark CO contours (shown as blue in the online version) superposed on the SCUBA-2 map indicate the extent of the blueshifted outflow. Contours run from 1.5 to $4.3 \mathrm{~K} \mathrm{~km} \mathrm{~s}^{-1}$ in steps of $0.4 \mathrm{~K} \mathrm{~km} \mathrm{~s}^{-1}$. Dashed white contours, running from 2 to $8 \mathrm{~K} \mathrm{~km} \mathrm{~s}^{-1}$ in steps of $2 \mathrm{~K} \mathrm{~km} \mathrm{~s}^{-1}$, and the small core of solid contours ranging from 10.0 to $10.3 \mathrm{~K} \mathrm{~km} \mathrm{~s}^{-1}$ show the emission about the systemic velocity of the cloud as explained in the text.

(A color version of this figure is available in the online journal.)

summing the source flux in the aperture and subtracting the sky flux. Since there is another relatively bright source in close proximity to A7, we were unable to use an annulus surrounding the aperture for sky measurements (in this case the sky refers to the faint extended emission surrounding the sources). Instead, several measurements of the surrounding faint extended (and variable) emission were obtained in an aperture the same size as the object photometry aperture, averaged to obtain a mean background for each source, and subtracted from the source photometry measurements. The uncertainties were obtained by adding in quadrature the uncertainty of the source flux and the standard deviation of the mean of the sky measurements (the non-uniformity of the background emission dominates other sources of uncertainty). Finally, the values were multiplied by the area of a pixel to obtain the flux densities in Jy. The resulting values are given in Table 3 .

The continuum flux density from heated dust may be contaminated by ${ }^{12} \mathrm{CO}$ line emission since the $J=3-2$ transition at $345.8 \mathrm{GHz}$ lies within the $850 \mu \mathrm{m}$ band. The amount of contamination can range from little to a few tens of a percent depending on factors such as the presence of strong $\mathrm{CO}$ outflows. 


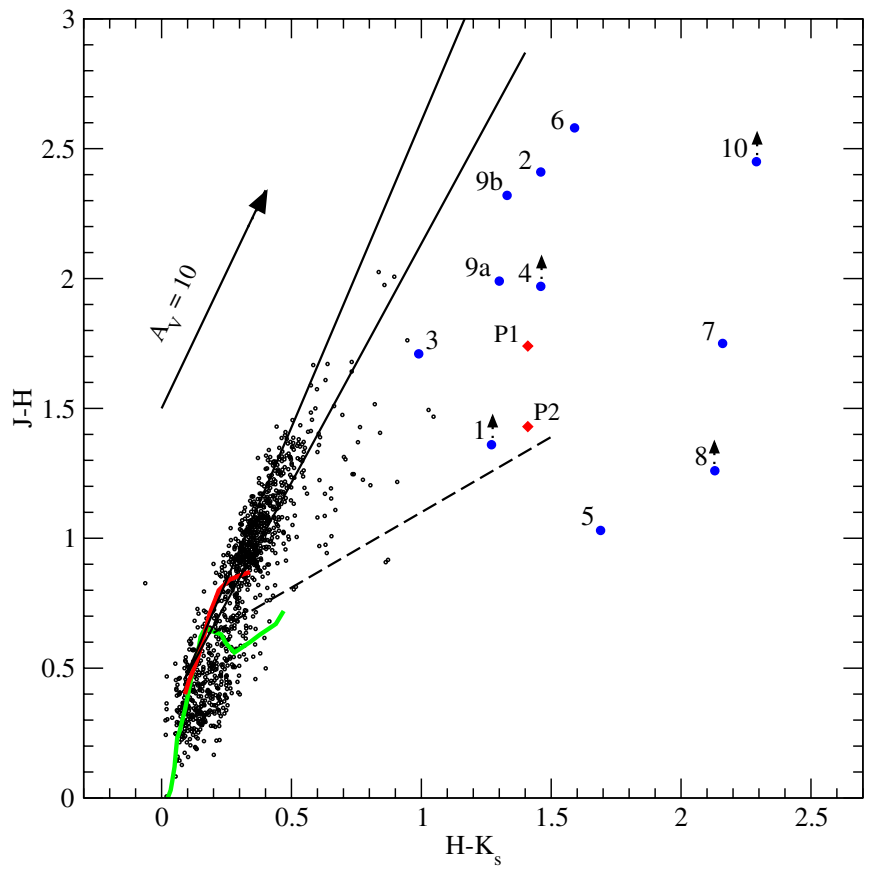

Figure 3. Color-color diagram for 1055 stars (small black circles) in the vicinity of Berkeley 59. Association objects are indicated by the large filled circles (colored blue in the online version) and labeled according to Table 2 (the prefix "A" has been omitted for clarity). Pillar objects are denoted by filled diamonds (red in the online version). The superposed lines and arrows beside some of the data points are described in the text.

(A color version of this figure is available in the online journal.)

Drabek et al. (2012) calculated conversion factors to convert $\mathrm{CO}$ integrated-intensity maps in units of $\mathrm{K} \mathrm{km} \mathrm{s}^{-1}$ to maps in units of mJy beam ${ }^{-1}$ to provide a direct comparison of flux densities between the SCUBA-2 and HARP maps. The value of the conversion factor depends on weather band: the appropriate conversion factor of $0.70 \mathrm{mJy}^{\text {beam }}{ }^{-1}$ per $\mathrm{K} \mathrm{km} \mathrm{s}^{-1}$ was applied to the HARP maps. The resulting flux densities in every pixel of the new maps were a fraction of a percent compared to the flux densities in the $850 \mu \mathrm{m}$ map; thus, a correction for $\mathrm{CO}$ line emission was not necessary for this part of the analysis. As a further check, aperture photometry of the apparent sources was also performed using a 14 arcsec diameter aperture on both maps, corrected for the size of the beam, and the resulting values compared. Again, the HARP flux density was a fraction of a percent of the $850 \mu \mathrm{m}$ flux density (the values were similar to what Drabek et al. 2012 obtained for some regions in their study).

\section{ANALYSIS AND RESULTS}

\subsection{Infrared Color-Color Diagrams and Reddening}

Straižys \& Laugalys (2008, and references therein) used data extracted from the $2 \mathrm{MASS}, I R A S$, and $M S X$ databases to produce color-color diagrams and search for infrared-excess objects. They also used these data to determine the reddening line for the regions studied. As a first step, we followed their procedure to construct a $J-H, H-K_{s}$ diagram and verify which objects (if any) exhibit IR excesses. The 2MASS Point Source Catalogue was searched for stars in a $1 \mathrm{deg}^{2}$ field centered on Berkeley 59 having photometric magnitude uncertainties in $J, H$, and $K_{s}$ less than 0.03. The resulting color-color diagram is shown in Figure 3 . The majority of the points in the diagram are typical field stars of various spectral classes and reddenings, while the reddest stars are predominantly $\mathrm{K}$ and $\mathrm{M}$ giants. Also shown are the intrinsic 2MASS relations for dwarfs and giants (solid green and red lines, respectively, in the online version) from Straižys \& Laugalys (2009), the reddening line for the region (described below, and indicated on the diagram by solid black lines), and the T Tauri locus (Meyer et al. 1997), which is indicated by the black dashed line. The association and pillar objects are denoted by large filled (blue) circles and (red) diamonds, respectively. The vector indicating a visual extinction of $10 \mathrm{mag}$ is shown as well, for the mean value of the slope of the reddening line (described below). Note that A1, A4, A8, and A10 were undetectable on the 2MASS $J$-band image; hence, their $J-H$ colors are "blue limits." Small arrows indicating the direction in which the colors would change for positive detections in $J$ are shown in Figure 3.

The reddening law is typically a weighted function of the size and metallicity of the grains across the entire sight line and distance covered. Stars beyond foreground clouds will have a superposition of the grain distributions. In this case, given the Galactic location of Berkeley 59 (in the outer disk and above the Galactic plane at $5^{\circ}$ ), it is likely that the primary source of dust grains affecting the extinction is associated with the cluster. The extinction program DUST ${ }^{6}$ was used to determine a mean color excess $\left\langle E_{B-V}\right\rangle=5.63 \pm 1.76$, resulting in a range of visual extinction $A_{V}$ from 12 to $23 \mathrm{mag}$. Red giant clump stars possess intrinsic colors of $(J-H)_{0}=0.46$ and $\left(H-K_{s}\right)_{0}=0.09$, as determined by Straižys \& Laugalys (2009) using 2MASS data; a value of $A_{V}=12$ mag results in a minimum color $J-H=1.4$. There are 15 field stars that have colors redder than this; they were used with the intrinsic colors to calculate color excess ratios, which were averaged to yield $E(J-H) / E\left(H-K_{s}\right)=2.10 \pm 0.26$. This result is similar to what V. Straižys (2012, private communication) found for regions of the Galaxy close to this one in Galactic longitude and latitude. The two extremes of the ratio (1.84 and 2.36) correspond to the slope of the reddening lines given in Figure 3 as solid black lines. It should be noted that the reddening line deviates from a straight line at large color excess $\left(E\left(H-K_{s}\right)>1\right.$; Straižys \& Lazauskaite 2008), but since the color excesses here were less than 1 , we have not considered this effect further, other than to use the uncertainty in the reddening line slope to verify that the objects that possess IR excesses do not change with the change in slope.

Most of the association and pillar objects appear to be potential YSOs; however, it is unwise to rely solely on nearIR magnitudes and color-color diagrams for identification of such sources, since other highly reddened objects are found in that region as well. Additional data are required in order to separate actual YSOs from extragalactic objects and latetype reddened stars. Early evidence of this was revealed by Strom et al. (1989) in their study of the L1641 molecular cloud. Confirmation of what they termed "positional coincidences" between the IRAS and near-IR detections, as well as the ability to separate true YSOs from normal stars and non-stellar objects, was possible only when spectra of those sources also present at optical wavelengths were combined with SEDs produced from the optical and near-IR data. Straižys \& Laugalys (2007) noted this as well in their study of the Camelopardalis region: a $J-H, H-K_{s}$ diagram revealed extensive contamination by non-YSOs, which were flagged only by the analysis of IRAS colors, where available.

\footnotetext{
6 http://irsa.ipac.caltech.edu/applications/DUST/
} 
Table 5

Results: SED Fitting (Best-fitting Model) and Spectral/Evolutionary Class Determinations

\begin{tabular}{|c|c|c|c|c|c|c|c|c|c|c|c|c|}
\hline $\begin{array}{l}\text { Figure } \\
\text { ID }\end{array}$ & $\begin{array}{c}M_{*} \\
\left(M_{\odot}\right)\end{array}$ & $\begin{array}{c}T_{*} \\
(\mathrm{~K})\end{array}$ & $\begin{array}{l}M_{\text {disk }} \\
\left(M_{\odot}\right)\end{array}$ & $\begin{array}{c}R_{\mathrm{disk}}^{\min } \\
(\mathrm{au})\end{array}$ & $\begin{array}{c}\dot{M}_{\mathrm{disk}} \\
\left(M_{\odot} \mathrm{yr}^{-1}\right)\end{array}$ & $\begin{array}{c}\dot{M}_{\mathrm{env}} \\
\left(M_{\odot} \mathrm{yr}^{-1}\right)\end{array}$ & $\begin{array}{c}\theta_{\text {cavity }} \\
\left({ }^{\circ}\right)\end{array}$ & $\begin{array}{c}A_{V} \\
(\mathrm{mag})\end{array}$ & $\begin{array}{c}d \\
(\mathrm{pc})\end{array}$ & Stage & $\alpha$ & Class \\
\hline $\mathrm{A} 2$ & 2.3 & 7860 & $1.6 \mathrm{E}-05$ & 0.41 & $9.0 \mathrm{E}-11$ & 0 & $\cdots$ & 16.9 & 851 & II/III & -0.02 & $\mathrm{f}(\mathrm{G}), \mathrm{f} / \mathrm{II}(\mathrm{K})$ \\
\hline A6 & 2.6 & 10900 & $1.3 \mathrm{E}-05$ & 0.49 & $4.5 \mathrm{E}-12$ & 0 & $\cdots$ & 16.6 & 977 & III & -0.29 & $\mathrm{f}(\mathrm{G}), \mathrm{II}(\mathrm{K})$ \\
\hline A7 & 1.2 & 4190 & $4.2 \mathrm{E}-02$ & 1.85 & 4.4E-08 & $3.9 \mathrm{E}-05$ & 18 & 14.7 & 708 & I & 1.55 & $\mathrm{I}(\mathrm{G}, \mathrm{K})$ \\
\hline $\mathrm{A} 7 *$ & 1.6 & 4270 & $5.0 \mathrm{E}-03$ & 2.54 & $1.4 \mathrm{E}-08$ & $3.3 \mathrm{E}-05$ & 28 & 14.2 & 955 & I & 1.55 & $\mathrm{I}(\mathrm{G}, \mathrm{K})$ \\
\hline A10 & 0.4 & 3500 & $5.6 \mathrm{E}-04$ & 0.15 & 4.9E-08 & $6.9 \mathrm{E}-05$ & 4 & 14.1 & 776 & I & 0.26 & f $(\mathrm{G}), \mathrm{I}(\mathrm{K})$ \\
\hline $\mathrm{P} 1$ & 4.5 & 9090 & $2.4 \mathrm{E}-06$ & 1.21 & $3.4 \mathrm{E}-12$ & $6.4 \mathrm{E}-08$ & 33 & 6.9 & 912 & II/III & 0.30 & $\mathrm{f}(\mathrm{G}), \mathrm{f} / \mathrm{II}(\mathrm{K})$ \\
\hline
\end{tabular}

Note. A7* refers to the model run that did not contain the SCUBA- 2 data, $\mathrm{f}=$ flat, $\mathrm{G}=\mathrm{Greene}$, and $\mathrm{K}=\mathrm{Koenig}$.

The use of both near- and mid-IR data may alleviate some of the difficulties mentioned above. For example, Allen et al. (2004), Robitaille et al. (2008), and Gutermuth et al. (2009) have shown using models and data from the Infrared Array Camera on the Spitzer Space Telescope that it is possible to use mid-IR color-color diagrams to distinguish between YSOs and extragalactic/reddened Galactic objects, and that the YSOs themselves may be separated into their different evolutionary stages. This method was adapted by Koenig et al. (2012), who used WISE colors and 2MASS $K_{s}$ magnitudes to classify the YSOs and set boundaries within which extragalactic objects may be found (and thus rejected from the sample; see Figures 7, 9, and 11 in that work).

According to the WISE colors computed from the magnitudes in Table 3, objects A7 and A10 fall solidly into Class I, while A2 and P1 are borderline between flat and Class II and A6 is Class II. These classifications are listed in the last column of Table 5 and denoted by "f" for flat and "K" for Koenig's method.

A classification scheme describing the stage of evolution of YSOs based on the slope, $\alpha$, of the SED between 2.2 and $22 \mu \mathrm{m}$ was first devised by Lada (1987) and revised by Greene et al. (1994). In the revised scheme, Class I, flat, Class II, and Class III sources have SED slopes of $\alpha>0.3,0.3>\alpha \geqslant-0.3$, $-0.3>\alpha \geqslant-1.6$, and $\alpha<-1.6$, respectively, and correspond to protostars possessing infalling envelopes (Class I and flat), pre-main-sequence stars with optically thick disks (Class II), and diskless pre-main-sequence stars (Class III). Evolutionary class determinations and spectral index $(\alpha)$ values of the sources in this work are presented in the last two columns of Table 5, where $\mathrm{G}$ represents the method of Greene et al. (1994). Each value of $\alpha$ was calculated using $K_{s}$ and $W 4$ (except for A6; $W 3$ was used since the magnitude at $W 4$ was an upper limit) and the equation given in Greene et al. (1994). Uncertainties in the observed and dereddened colors were calculated by adding the individual uncertainties (magnitudes for observed colors, and observed colors and extinctions) in quadrature. We have chosen to calculate $\alpha$ using the data at 2.2 and $22 \mu \mathrm{m}$ rather than quoting the slope of the line corresponding to a least-squares fit of all the data, since the former provides a unique value for each protostar. For the most part these agree with the class determinations obtained from the WISE colors.

\subsection{Spectral Energy Distributions}

Robitaille et al. (2006) developed a grid of model SEDs to be used for fitting to data, in order to constrain the visual extinction and distance to the source and determine various source parameters. They found that the spectral index and color of a YSO are not always indicative of its stage of evolution, and therefore they adopted a different classification "Stage" scheme that relates the physical properties of the YSO as determined from the best-fitting SED models, rather than the slope of the SED at IR wavelengths. Their classification scheme is used in conjunction with the Class scheme in order to describe an object unambiguously.

The near- and mid-IR data for A2, A6, A7, A10, and P1 were used to construct SEDs and fit models, with the inclusion of the 450 and $850 \mu \mathrm{m}$ flux densities for A7 and A10. The starting range for the distance was given according to the distance estimate of $880 \pm 40 \mathrm{pc}$ for Berkeley 59 by Majaess et al. (2008), who used MacConnell's (1968) $U B V$ photometry to determine the mean cluster color excess and their own $B V$ photometry to produce a color-magnitude diagram and corresponding ZAMS fit. Starting extinctions were as determined in Section 3.1; thus, in both regions the distance ranged from 0.7 to $1.0 \mathrm{kpc}$, while the extinction ranged from 12 to $23 \mathrm{mag}$ and 5 to $23 \mathrm{mag}$ for the association and pillar, respectively. The lower limit for the pillar was altered to reflect P1's partial visibility at optical wavelengths. Figure 4 shows the SEDs for the objects listed above. Note that although profile-fitting photometry was conducted for the near- and mid-IR data used in the models, the apertures used in the model data pertain to those used by the 2MASS and WISE pipelines in converting their profile-fitting photometry to standard aperture magnitudes, namely, $4^{\prime \prime}$ for 2MASS, 7".5 for $W 1, W 2$, and $W 3$, and $15^{\prime \prime}$ for $W 4$, since we had tied our photometry to those photometric systems. An aperture of $30^{\prime \prime}$ was used for MSX.

Although there are 14 parameters used in the YSO model fits, Table 5 contains values for those that are most applicable to younger objects and that most affect the 1-8 $\mu \mathrm{m}$ fluxes. Column headings pertaining to the models include the object ID number, protostellar mass, protostellar temperature, disk mass, the inner radius of the disk, disk accretion rate, envelope accretion rate, opening angle of the envelope cavity carved out by strong outflows, and the evolutionary stage as deduced from the models. The range in values for the 10 best-fitting models is presented in Table 6.

It should be noted that the SCUBA-2 filters have not yet been added to the SED fitting tool. A comparison of the filter profiles found on the JCMT Web site indicated that the $850 \mu \mathrm{m}$ SCUBA2 filter is wider than its predecessor by about $15 \mu \mathrm{m}$, while the $450 \mu \mathrm{m}$ SCUBA-2 filter is narrower than the corresponding SCUBA filter by $8 \mu \mathrm{m}$. Both the broadband SCUBA filter setting and the monochromatic wavelength setting (set to the central wavelengths of the filters) were used in the fits to the A7 and A10 data; in both cases the same best-fitting model was chosen.

The tool uses evolutionary tracks along with stellar masses to obtain the stellar radii and temperatures, which means that an estimate of the protostellar age is also provided. As expected, it 

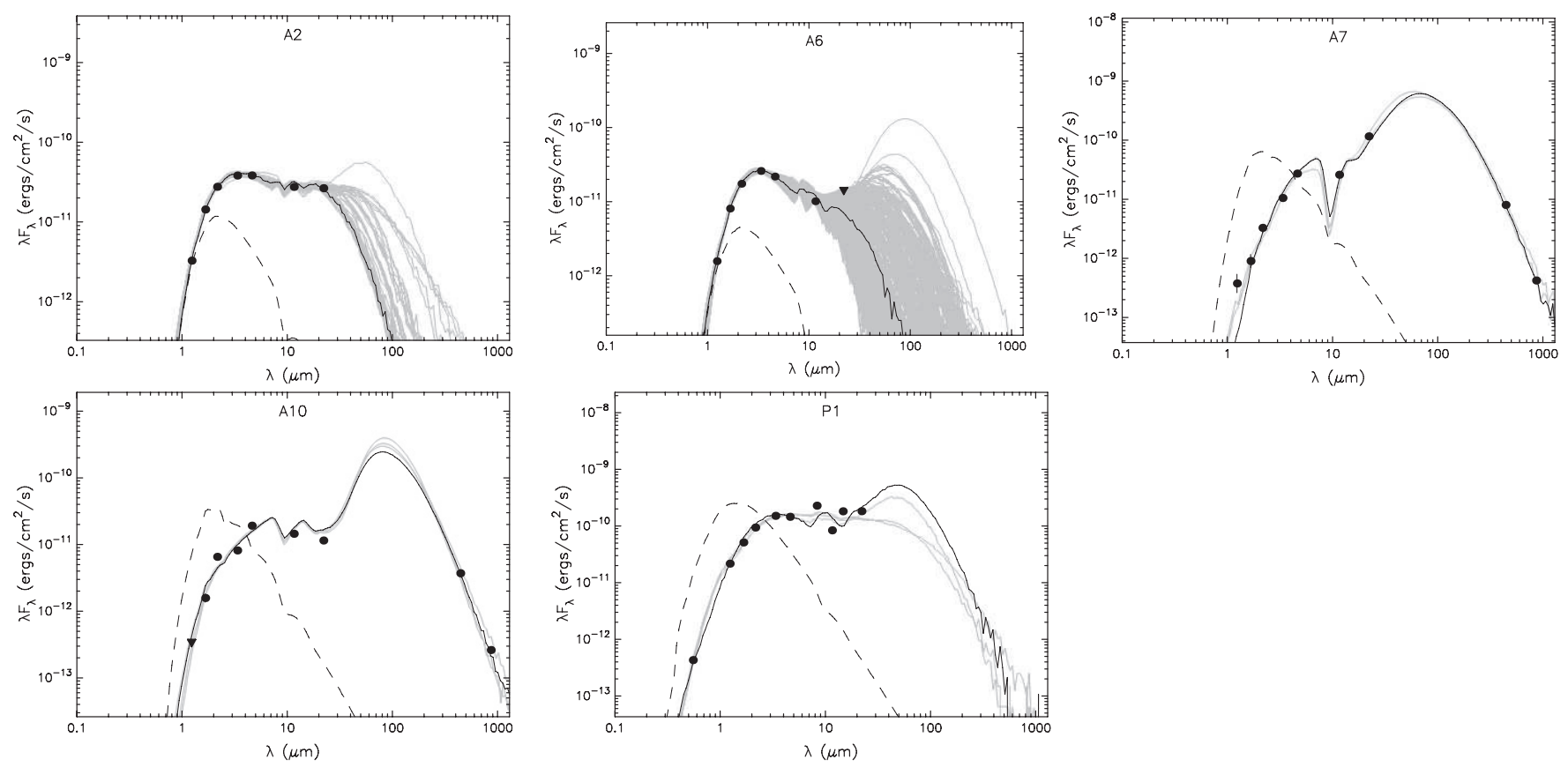

Figure 4. SEDs of protostars using near- and mid-IR data. In each diagram, the solid black lines refer to the best-fitting models, while the gray lines correspond to models that fit the data equally well. The dashed line refers to the SED of the best-fitting stellar photospheric model.

Table 6

SED Fitting Results: Ranges for the 10 Best Fits

\begin{tabular}{|c|c|c|c|c|c|c|c|}
\hline Figure ID & $\begin{array}{c}M_{*} \\
\left(M_{\odot}\right)\end{array}$ & $\begin{array}{c}T_{*} \\
(\mathrm{~K})\end{array}$ & $\begin{array}{l}M_{\text {disk }} \\
\left(M_{\odot}\right)\end{array}$ & $\begin{array}{c}R_{\text {disk }}^{\min } \\
\text { (au) }\end{array}$ & $\begin{array}{c}\dot{M}_{\mathrm{disk}} \\
\left(M_{\odot} \mathrm{yr}^{-1}\right)\end{array}$ & $\begin{array}{c}\dot{M}_{\mathrm{env}} \\
\left(M_{\odot} \mathrm{yr}^{-1}\right)\end{array}$ & $\begin{array}{c}\theta_{\text {cavity }} \\
\left({ }^{\circ}\right)\end{array}$ \\
\hline $\mathrm{A} 2$ & $2.3-3.2$ & $6500-11300$ & 1.6E-05-3.7E-03 & $0.41-1.2$ & $9.0 \mathrm{E}-11-1.1 \mathrm{E}-08$ & 0 & $\ldots$ \\
\hline A6 & $2.6-3.3$ & $7440-12300$ & 2.2E-07-2.8E-05 & $0.49-0.8$ & 4.5E-12-1.6E-10 & 0 & $\ldots$ \\
\hline A7 & $0.3-4.8$ & $3430-4870$ & $1.2 \mathrm{E}-02-1.0 \mathrm{E}-01$ & $0.21-9.5$ & 1.2E-08-4.6E-06 & $2.5 \mathrm{E}-05-1.6 \mathrm{E}-04$ & $17-39$ \\
\hline $\mathrm{A} 7 *$ & $0.3-1.9$ & $3430-4300$ & $2.0 \mathrm{E}-03-9.2 \mathrm{E}-02$ & $0.21-2.7$ & 3.9E-09-4.6E-06 & 7.2E-06-6.1E-05 & $17-34$ \\
\hline A10 & $0.4-1.0$ & $3500-4100$ & 4.5E-04-6.6E-02 & $0.15-1.6$ & 8.0E-09-9.7E-07 & $2.4 \mathrm{E}-05-2.0 \mathrm{E}-04$ & $3-31$ \\
\hline $\mathrm{P} 1$ & $2.8-4.5$ & $9090-13600$ & 2.4E-06-4.2E-02 & $1.2-2.9$ & 3.4E-12-5.5E-07 & $0-6.4 \mathrm{E}-08$ & $\cdots(42)$ \\
\hline
\end{tabular}

Notes. Only one P1 model produced a non-zero envelope accretion rate and defined cavity opening angle, reflecting the non-zero entries above.

was found that the age correlated with evolutionary class, with values ranging from 0.001 to over $4 \mathrm{Myr}$ for the association objects, and $0.8 \mathrm{Myr}$ for P1. The tool also provides estimates of the distance and interstellar extinction. These values are given in Table 5.

\subsection{The Emission-magnitude Diagram}

As mentioned in the Introduction, the presence of $\mathrm{Br} \gamma$ emission is a characteristic of CTTSs. Several methods exist to determine if objects exhibit this type of emission, including image subtraction and the construction of an emission-magnitude diagram (EMD). The first technique was used by Varricatt et al. (2010), who obtained broad- and narrowband UKIRT data to study outflow candidates and found that some of their sources exhibited excess $\mathrm{Br} \gamma$ emission. In this technique, a flux-scaled continuum image is subtracted from a narrowband $\mathrm{Br} \gamma$ image. This method is subject to difficulties involving background subtraction, image registration, focusing, and point-spread function changes across the field and between images. EMDs have been used successfully to separate Wolf-Rayet stars from ordinary stars (Shara et al. 2009) on the basis of $\mathrm{He} \mathrm{I}+\mathrm{Br} \gamma$ emission lines that appear at wavelengths covered by the narrowband $\mathrm{Br} \gamma$ filter. To our knowledge, the latter method has not yet been used to search for CTTSs.
Initially the first technique was attempted, using the (skysubtracted) $\mathrm{Br} \gamma$ and CONT2 images. The images were convolved with a $3 \sigma$ Gaussian to smooth the background variations and examined to determine if any flux scaling was necessary by calculating integrated fluxes in a 7 arcsec radius aperture centered on bright, uncrowded stars in regions free from contamination. Care was taken to ensure that stars exhibiting IR excesses were not included in the list. For each of 219 stars selected, the ratios of the CONT2 to $\mathrm{Br} \gamma$ fluxes were calculated and averaged to yield a mean ratio of $0.98 \pm 0.07$. Since no flux scaling was necessary (based on the calculated ratio), the CONT2 and $\operatorname{Br} \gamma$ images were spatially aligned and subtracted. The subtracted image revealed inconsistent under- and over-subtraction of ordinary stars, as well as a spatially offset subtraction that was acute for stars around the edges of the frame, suggesting a spatial scaling/rotation issue between the two images despite a good alignment between the images at the image center. After unsuccessful attempts to correct for this effect, the method was abandoned and the latter one pursued.

An EMD was constructed following the description by Shara et al. (2009). A star having excess $\mathrm{Br} \gamma$ emission will be brighter than the continuum measured at that wavelength. To facilitate the comparison, a continuum magnitude at the central wavelength of the $\mathrm{Br} \gamma$ filter was estimated by linear interpolation between 


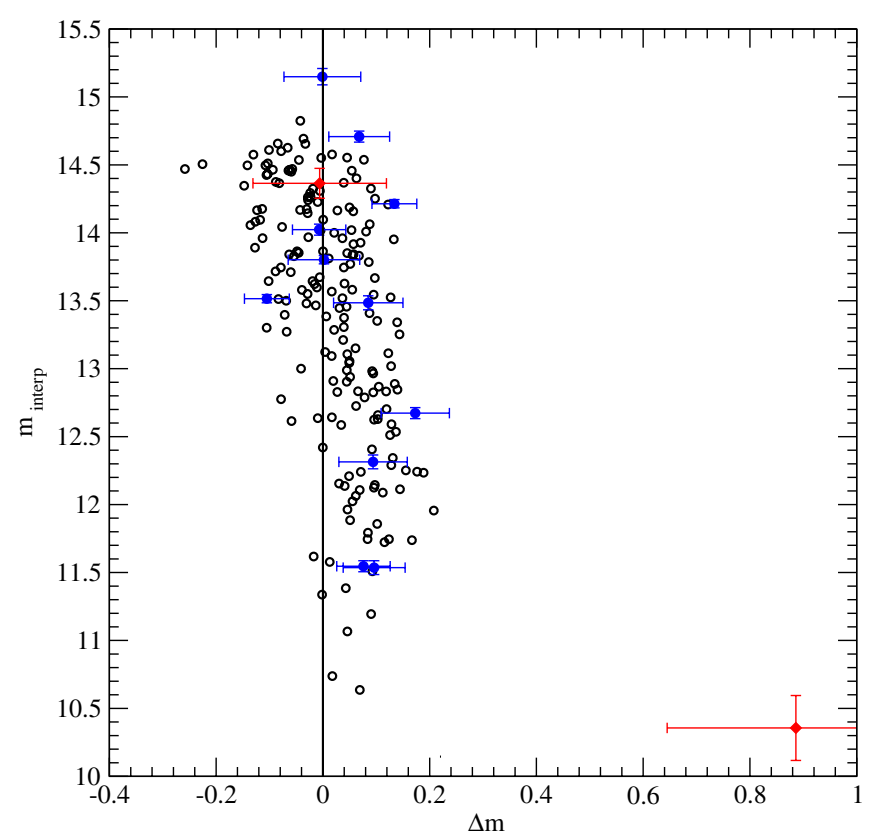

Figure 5. Emission-magnitude diagram of typical field stars (open circles), association objects (filled circles, blue in the online version), and pillar objects (filled diamonds, red in the online version). The interpolated continuum magnitude at the wavelength of the $\mathrm{Br} \gamma$ filter is plotted against the emission magnitude. The determination of the error bars is discussed in the text. P1 is located at an emission magnitude $\Delta m$ of $0.89 \mathrm{mag}$.

(A color version of this figure is available in the online journal.)

the two continuum measurements. Next, the $\mathrm{Br} \gamma$ magnitude was subtracted from this value to obtain an emission magnitude $\Delta m$. A plot of interpolated continuum magnitude versus $\Delta m$ for ordinary stars shows data points clustering around $\Delta m=0$. Any outliers on the positive side of $\Delta m$ are potential excess emission objects. Since ordinary stars should have flat continua in the wavelength range between the two continuum images, and stars having poor photometry in one of the images could muddy the results, only those stars having flat or nearly flat slopes in the interpolation step were retained. These stars thus set the magnitude of the scatter about $\Delta m=0$. The same procedure was followed for the association and pillar objects.

Figure 5 plots the results. The association objects are indicated by filled (blue) circles, while the pillar objects appear as filled (red) diamonds. Ordinary stars are denoted by open circles. Error bars for the YSOs are shown as well and were determined for $m_{\text {interp }}$ by using standard uncertainty propagation rules with the relevant magnitudes and equations for slope and intercept. These were added in quadrature with the $\mathrm{Br} \gamma$ photometric uncertainties to obtain uncertainties for $\Delta m$. Note that the stars deviate from $\Delta m=0$ at brighter interpolated magnitudes. This could be due to difficulties in the calibration of CONT1, but since both sets of data (field and YSO) exhibited the same deviation, it was considered to be a non-issue in the scope of this analysis.

Shara et al. (2009) calculated the standard deviation in $\Delta m$ for each 1 mag bin of interpolated magnitude and assumed that any object possessing a $\Delta m 5 \sigma$ from the mean of zero was a Wolf-Rayet candidate. Since the stars here were selected on the basis of the 2MASS photometry, and none were fainter in $K_{S}$ than about $15 \mathrm{mag}$, the dispersion in each magnitude bin did not vary much: standard deviations of the emission magnitudes in each $1 \mathrm{mag}$ bin were $0.06,0.06,0.07$, and $0.08 \mathrm{mag}$ for the bins $11-12,12-13,13-14$, and 14-15 mag, respectively. Figure 5

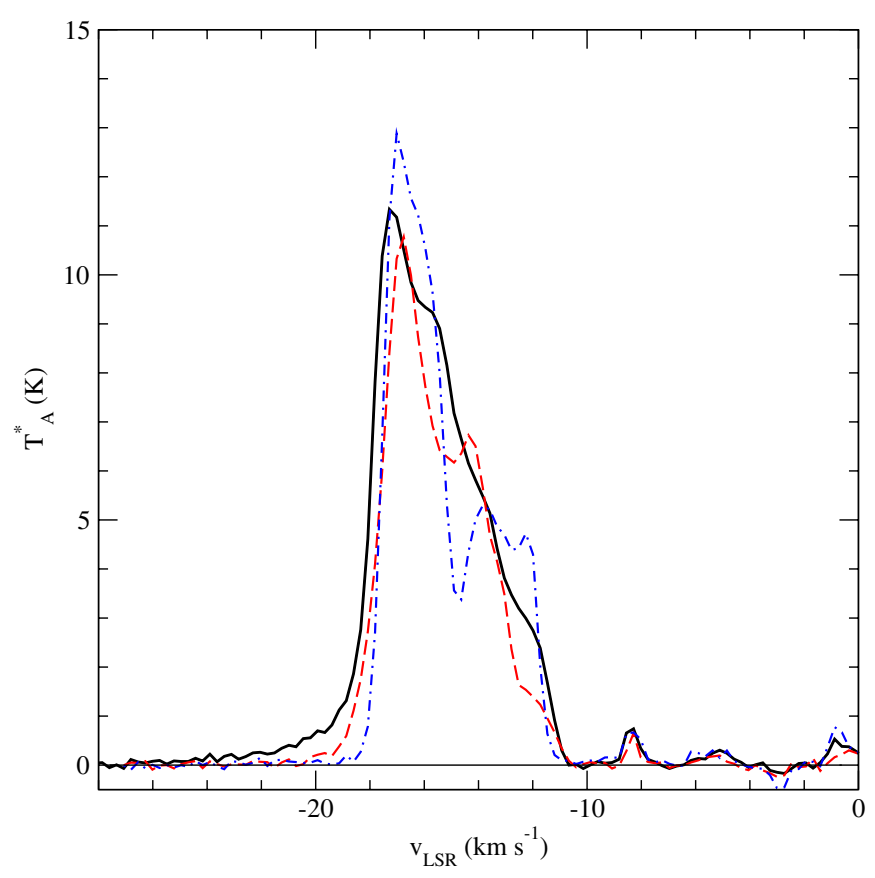

Figure 6. CO spectra of the association. The heavy black line represents a spatially averaged spectrum from the center of the map. The dashed (red in the online version) and dot-dashed (blue in the online version) lines correspond to spatially averaged spectra obtained from the northernmost and southeasternmost, pixels, respectively. Smaller peaks, due to superposed clouds of different velocities, are discussed in the text.

(A color version of this figure is available in the online journal.)

indicates that $\mathrm{P} 1$, appearing in the lower right corner, exhibits excess $\mathrm{Br} \gamma$ emission according to the $5 \sigma$ criterion, even if the apparent trend toward larger $\Delta m$ continues: at the magnitude of $\mathrm{P} 1$, the value of $\Delta m$ for field stars would reach $\sim 0.2 \mathrm{mag}$, leading to a "corrected" $\Delta m$ for P1 close to $0.7 \mathrm{mag}$. Assuming the same standard deviation of about $0.07 \mathrm{mag}$, the emission magnitude of P1 is still greater than $5 \sigma$. This result, as well as a tie-in to various $\mathrm{H} \alpha$ studies as a check on the validity of the method, will be explored in Section 4.2.

\subsection{CO Emission}

$\mathrm{CO}$ emission is pervasive throughout the Cep OB4 region, and it was our intent to map the area immediately surrounding the association and pillar in order to determine whether outflows were present. Spectra were plotted on a per-pixel basis to identify possible outflows, and channel maps were produced to narrow down the velocity ranges. Typical methods for determining outflow velocity ranges involve studying the extended wings of line profiles and selecting cut-off values based on, for example, a certain distance in velocity from the peak velocity of the line and a certain $\sigma_{\text {rms }}$ above background (Curtis et al. 2010). Since it was difficult to identify the line centers, we were inclined to follow a more subjective approach by manually inspecting the channel maps and spectra to select the velocity ranges (note that position-velocity diagrams were also generated, but they were not very useful since the detected outflow was weak and there is substantial self-absorption present in the data). Integrated-intensity maps over the velocity range (down to $T_{\mathrm{A}}^{*}=0$ ) were produced based on the by-eye determination of the outflow emission.

Figure 6 shows a selection of spectra of the $\mathrm{CO}$ emission for the association. The heavy black line corresponds to a spectrum obtained by spatially averaging the central 

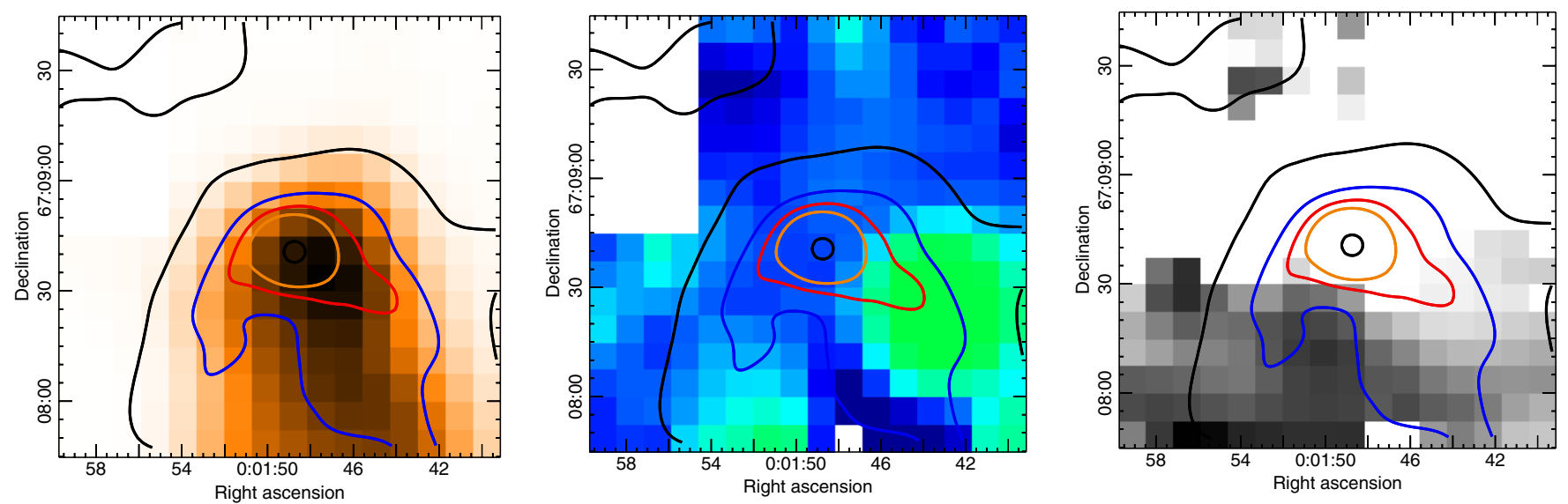

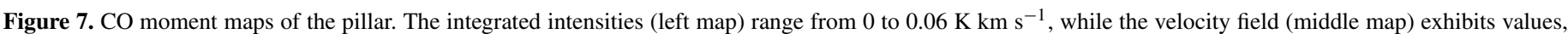

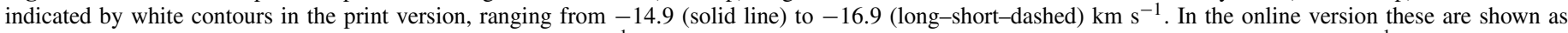

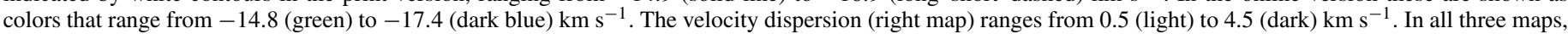

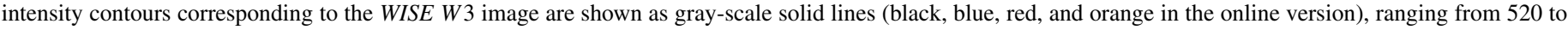
1120 data numbers (DN); the small circle denotes the position of P1.

(A color version of this figure is available in the online journal.)

25 pixels of the map in order to emphasize the blue line wing. Some individual pixel spectra (predominantly at the northernmost, southernmost, and eastern edges of the map) exhibited double peaks of varying morphology. The dashed line corresponds to a spectrum obtained by spatially averaging the northernmost two rows of pixels. The velocity of the redward peak remained fixed at $-14.4 \mathrm{~km} \mathrm{~s}^{-1}$, while the blueward peak shifted between -16.7 and $-17.3 \mathrm{~km} \mathrm{~s}^{-1}$, depending on the pixel location. The dip between the two peaks moved between -15 and $-16.5 \mathrm{~km} \mathrm{~s}^{-1}$. The dot-dashed line represents a spectrum obtained by spatially averaging a selection of pixels in a $5 \times 5$ square in the southeasternmost corner of the map. This was the only location where the dip (which remained fixed at $-15 \mathrm{~km} \mathrm{~s}^{-1}$ ) reached such a low antenna temperature. Line widths for the individual pixel spectra ranged from 2 to $5 \mathrm{~km} \mathrm{~s}^{-1}$ depending on location.

Since any redshifted outflow that exists is obscured by selfabsorption, we focused on the blueshifted outflow. On the basis of the black-line spectrum given in Figure 6 and the channel maps, velocities spanning the range -27 to $-18.6 \mathrm{~km} \mathrm{~s}^{-1}$ were selected as limits for the generation of the integrated-intensity map. Contours from this map are superposed on the SCUBA-2 $450 \mu \mathrm{m}$ map given in Figure 2 and indicated by solid lines (blue in the online version). Contours run from 1.5 to $4.3 \mathrm{~K} \mathrm{~km} \mathrm{~s}^{-1}$ in steps of $0.4 \mathrm{~K} \mathrm{~km} \mathrm{~s}^{-1}$. Also plotted are white contours about the systemic velocity, which were derived from an integratedintensity map spanning the velocity range -18 to $-15 \mathrm{~km} \mathrm{~s}^{-1}$. These contours run from 2 to $8 \mathrm{~K} \mathrm{~km} \mathrm{~s}^{-1}$ in steps of $2 \mathrm{~K} \mathrm{~km}$ $\mathrm{s}^{-1}$ and are indicated by dot-dashed for the two lowest contours and then short- and long-dashed for the next two. The small cluster of solid contours runs from 10.0 to $10.3 \mathrm{~K} \mathrm{~km} \mathrm{~s}^{-1}$ and marks the location of the peak of the spectral line at $-17 \mathrm{~km}$ $\mathrm{s}^{-1}$ (see Figure 6). The contours reflect the relative size of the jiggle map footprint to the SCUBA-2 image.

${ }^{12} \mathrm{CO}(J=3-2) \mathrm{CO}$ spectra of the pillar exhibit line widths of at least $4 \mathrm{~km} \mathrm{~s}^{-1}$, with the bulk due to self-absorption in various areas across the CO map. The line is strong, with peak antenna temperature of just over $20 \mathrm{~K}$. As with the association data, spectra along various pixels were examined, and channel maps produced, to narrow down any outflow ranges. It was much more difficult to identify outflows in this region. Figure 7 presents the moment maps of the pillar. In all three maps, the black, blue, red, and orange contours refer to emission observed in the WISE $W 3$ image and range from 520 to 1120 data numbers (DN; in the print version the contours are represented by gray-scale shades, with DN increasing from the outermost contour to the innermost one). The small black circle, at $2000 \mathrm{DN}$, denotes the position of P1. The white region in the upper left corner corresponds to the part of the map not observed because of the dead receptor.

The integrated intensity map (left) was produced by collapsing the data cube in the velocity range -11 to $-19 \mathrm{~km} \mathrm{~s}^{-1}$. Map colors (gray-scale shades in the print version) range from 0 to $0.06 \mathrm{~K} \mathrm{~km} \mathrm{~s}^{-1}$. The strongest emission occurs near the location of P1 and extends down the center of the pillar, while the fainter emission at the edges of the pillar (as outlined by the W3 band contours) drops into the noise outside of the pillar's extent. The colors in the velocity field (middle map) correspond to velocities ranging from -14.8 (green) to -17.4 (dark blue) $\mathrm{km} \mathrm{s}^{-1}$. In the print version, these are indicated by white contours: solid, short-dashed, long-dashed, and short-long-dashed correspond to velocities from -14.9 to $-16.9 \mathrm{~km} \mathrm{~s}^{-1}$. The velocity field is not uniform and does not increase monotonically across the map in any direction. Velocities at the pillar's head correspond to the systemic velocity of $\sim-16 \mathrm{~km} \mathrm{~s}^{-1}$, while two other regions exhibit velocities at -15.2 (bottom of the map) and -14.8 (eastern edge of map) $\mathrm{km} \mathrm{s}^{-1}$. The velocity dispersion (right) ranges from 0.5 (light) to 4.5 (dark) $\mathrm{km} \mathrm{s}^{-1}$, with the largest values corresponding to the location of the non-systemic velocities.

In addition to the main $\mathrm{CO}$ emission, three other peaks occur at approximately $-8,-5$, and $-1 \mathrm{~km} \mathrm{~s}^{-1}$. A channel map of the association data from -8.8 to $1.2 \mathrm{~K} \mathrm{~km} \mathrm{~s}^{-1}$, in bins of $1 \mathrm{~K} \mathrm{~km} \mathrm{~s}^{-1}$, indicates that the first peak is present over the whole map, with integrated intensities in the first channel peaking at $0.75 \mathrm{~K} \mathrm{~km} \mathrm{~s}^{-1}$. The second peak is generally lower in intensity and concentrated in the southwest half of the map, with maximum integrated intensity of $0.5 \mathrm{~K} \mathrm{~km} \mathrm{~s}^{-1}$, while the third peak's emission is concentrated in the southeast half of the map. An integrated-intensity map over two channels (ranging from -1.7 to $0.2 \mathrm{~K} \mathrm{~km} \mathrm{~s}^{-1}$ ) displays a peak intensity of $0.9 \mathrm{~K} \mathrm{~km} \mathrm{~s}^{-1}$. This component is present in the vicinity of the pillar as well. 


\section{DISCUSSION}

\subsection{The SEDs}

According to Robitaille et al. (2006), Stage 0 and I objects possess substantial infalling envelopes and may have disks, Stage II YSOs possess optically thick disks and possibly the last remains of an infalling envelope, while Stage III objects possess optically thin disks. Those authors quantified their claim by an analysis of the mass ratio and envelope accretion rate: disks are optically thin for mass ratios $M_{\text {disk }} / M_{*}<10^{-5}$, and envelopes are optically thin for envelope accretion rates $\dot{M}_{\text {env }} / M_{*}<10^{-6} \mathrm{yr}^{-1}$. This section begins with a summary of the Class evolutionary system in this context and a discussion of the results of the SED fits. We caution that the values of the parameters are intended to be broad constraints only; since full wavelength coverage is lacking, it is not possible to constrain the values further.

A summary of the properties of Class I, flat, II, and III sources was given in Section 3.1. Class I sources also possess envelopes with bipolar cavities carved by strong outflows, with the cavity opening angle increasing as later Class I stages are reached; by Class II the envelope has dispersed, according to Whitney et al. (2003) and their radiative transfer models of protostars. In addition, the SED models show that varying inclinations of the disk can cause confusion between evolutionary classes. For example, an edge-on embedded protostar (viewing angle $i=87^{\circ}$ ) that normally would be classified as Class I may exhibit a negative slope between 2 and $10 \mu \mathrm{m}$ if its SED is dominated by either scattered light or an edge-on disk, and thus be classified as Stage II, whereas a pole-on embedded (Stage I) source may exhibit a flat SED, rather than a rising one (Robitaille et al. 2006, and references therein).

Object A7 is the only target with an SED that rises substantially in the mid-IR (an argument may be made that A10's SED rises as well, if one considers the overall trend from 3.4 to $22 \mu \mathrm{m})$. These objects are embedded, possess optically thick disks, and fall under Stage I on the basis of the model parameters. Their envelope accretion rates as a function of stellar mass exceed the boundary value of $10^{-6} \mathrm{yr}^{-1}$, which suggests they also possess optically thick accretion envelopes that make a significant contribution to the SED. The cavity opening angles are the smallest for these objects. It was mentioned earlier that the evolutionary class may not necessarily agree with the evolutionary stage, since the object can be described differently depending on the viewing angle of the best-fitting model. There are no such disparities with these sources. The best 10 models for each source all support a Stage I classification despite the range in viewing angles (inclination $i$ in the range $32^{\circ}-70^{\circ}$ for A7 and $18^{\circ}-63^{\circ}$ for A10; Robitaille et al. 2007 caution that even with full wavelength coverage, it is not always possible to constrain the inclination).

Further discussion of A7's SED is warranted. The model fit assumed that the submillimeter emission was associated with the suspected protostar. While the peak submillimeter emission is not necessarily going to coincide with the location of the object at other wavelengths, in this case the separation between the peak emission and the 2MASS position is rather large, at 8 arcsec. It is possible that the dust emission centered on 00:00:44.5, +67:32:55 and 00:00:42.2, +67:33:09 in Figure 2 is not related directly to A7. The YSOs in the region may be illuminating the dust, or (a more exciting possibility) the dust emission may be a consequence of even more deeply embedded Class 0 objects appearing only at submillimeter wavelengths and exhibiting no near- or mid-IR flux. If the latter is true, it indicates that star formation is occurring in the cloud, which is resulting in protostars spanning a broad range of evolutionary stages. Observations at millimeter wavelengths with higher resolution would reveal what is at the center of the dust emission, and what might be driving the weak $\mathrm{CO}$ outflow. Note that if the submillimeter data are removed from the SED and the model rerun, the values in Table 5 all change, but the conclusions regarding evolutionary class and stage remain the same. These new values are flagged in the table by a star next to the ID number.

Objects A2 and P1 possess nearly flat SEDs at IR wavelengths and are in Stage III according to the model parameters (the morphology of the SED suggests Stage II, hence the inclusion of both stages in Table 5). A2's low disk mass and zero accretion rate indicate that the disk is optically thin and the envelope has dissipated, implying that the object is more advanced in its evolution than A7 and A10. A breakdown of the contributions by the disk, envelope, and direct stellar flux reveals that the stellar flux is the dominant component. According to the model, P1's disk and envelope are optically thin, which makes physical sense if the source is evolved enough that little to no envelope remains, it is located at the edge of the cloud, and it is partially visible. These objects are more massive than A7 and A10, which supports their later evolutionary stages.

A6 possesses a decreasing slope beyond the near-IR wavelengths, and the sheer number of gray lines (which correspond to comparable model fits) in the top middle panel of Figure 4 indicates how difficult it was to obtain a single best-fitting model to these data, which is not surprising given that there is no anchor at long wavelengths. Any conclusions drawn from the models will be weak, but the downturn in the SED between 3.4 and $12 \mu \mathrm{m}$ is greater than that for the other models; thus, the classifications given in Table 5 indicate (at least tentatively) that this object probably is the most evolved.

The distances for the association objects average $830 \pm$ $120 \mathrm{pc}$. This value drops to $780 \pm 70 \mathrm{pc}$ if the distance of $A 6$ (the source with the least certain model solution) is omitted, which is fully $100 \mathrm{pc}$ smaller (but still within the uncertainties) than the distance of $880 \mathrm{pc}$ for Berkeley 59. Some of the discrepancy may arise from the value of the ratio of total to selective absorption chosen for each region. Majaess et al. (2008) found $R_{V}=2.8$ for the cluster, while the value of 3.1 was used by T. P. Robitaille (2013, private communication) in his SED tool. If the larger value of $R_{V}$ is used with Majaess et al.'s (2008) data, the distance to Berkeley 59 drops to just over $730 \mathrm{pc}$. Visual extinction and distance for P1 were 6.9 mag and $912 \mathrm{pc}$, respectively, the distance of which is substantially larger than the adjusted one for Berkeley 59. However, based on the agreement between the $\mathrm{CO}$ and cluster velocities, we are confident that the pillar is associated with the Berkeley 59 region.

\subsection{The EMD and $P 1$}

The EMD indicated that P1 is the only candidate CTTS on the basis of excess $\mathrm{Br} \gamma$ emission. As a further check, IPHAS was used to search for an occurrence of the source, since both $\mathrm{Br} \gamma$ and $\mathrm{H} \alpha$ emission is present in excess in CTTSs and P1 is low enough in Galactic latitude $(b=4.7)$ to have been included in the survey. Following the method of Barentsen et al. (2011), who used their data to devise a method for preferentially selecting T Tauri candidates, we selected objects in the field of Berkeley 59 having a high probability of being stellar and retained those whose magnitude errors were less than 0.04 and 


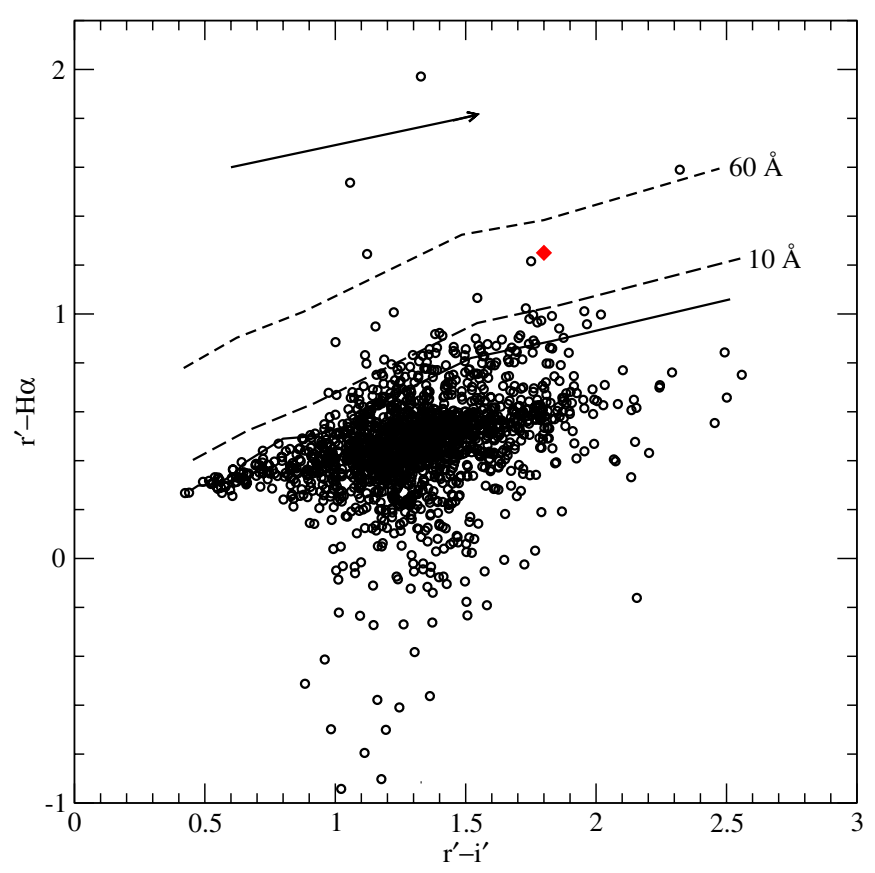

Figure 8. IPHAS color-color diagram of the field in the vicinity of Berkeley 59 Field stars are indicated by open circles, while P1 is plotted as a filled diamond (red in the online version). The solid, long-dashed, and short-dashed lines refer to the (unreddened) main sequence and two different amounts of excess $\mathrm{H} \alpha$ emission. The vector indicating the amount of visual extinction for Berkeley 59 corresponding to $\left\langle E_{B-V}\right\rangle=1.38$ is shown as well.

(A color version of this figure is available in the online journal.)

were fainter than $r^{\prime}=13$ mag. P1 was specifically selected as well. A color-color diagram of these data is shown in Figure 8 . Field stars are indicated by open circles, while P1 is denoted by a filled diamond (red in the online version) at $r^{\prime}-i^{\prime}=1.80, r^{\prime}-\mathrm{H} \alpha=1.25$. Also plotted are the unreddened main sequence (solid line) from Drew et al. (2005) and two lines indicating amounts of $\mathrm{H} \alpha$ emission corresponding to spectral line equivalent widths (EWs) of 10 and $60 \AA$ (long-dashed and short-dashed lines, respectively). The arrow indicates the visual extinction corresponding to the mean color excess of Berkeley $59\left(\left\langle E_{B-V}\right\rangle=1.38\right)$, assuming $R_{V}=3.1$. Note that P1 appeared twice in the catalog; its colors were obtained by averaging the two values for each color.

Figure 8 illustrates that P1 is a CTTS on the basis of its IPHAS colors. It lies well above the locus for ordinary stars (see, e.g., Figure 6 of Barentsen et al. 2011), and if its colors are dereddened, it falls just slightly above the line corresponding to emission having an EW of $60 \AA$. The CTTS classification is consistent with the results presented earlier regarding evolutionary status, and consistent with the object being faintly visible at optical wavelengths. In addition, the dereddened 2MASS colors place it in the region occupied by the T Tauri locus (see Figure 3).

At this point it should be stressed that while the IPHAS color-color diagram separates CTTSs from ordinary stars effectively, it also separates other $\mathrm{H} \alpha$ emitters with equal efficacy. This was explored in depth by Corradi et al. (2008) in their study of symbiotic stars (they also investigated the location on the IPHAS and 2MASS color-color diagrams of planetary nebulae, Be stars, and cataclysmic variables). After dereddening P1's colors and plotting the object on Corradi et al.'s (2008) Figures 2 and 5 (both 2MASS color-color diagrams), it was found that P1 fell in the region occupied by D-type symbiotic stars; however,
CTTSs are also found in that region on the 2MASS diagrams. Given the other evidence presented in this work, we are confident that P1 is a likely candidate for CTTS status, although a spectrum of the object is required to support this conclusion.

Muzerolle et al. (1998) investigated the relationship between $\mathrm{Br} \gamma$ emission line strength and mass accretion rate using several known CTTSs with varying accretion rates and found a strong correlation between accretion rate and line flux. Some of the stars in their study possessed low accretion rates, and accordingly the spectra exhibited no emission lines. This effect was supported by Barentsen et al.'s (2011) results. Returning to the EMD, line strengths in $\mathrm{Br} \gamma$ are only about $1 \%$ those of $\mathrm{H} \alpha$ (Osterbrock \& Ferland 2006; Beck et al. 2010), implying that unless the star is a strong emitter, the EMD technique would not be useful in selecting emission-line stars. A rough estimate of the minimum EW required for detection may be made using P1's data and the observed scatter of 0.07 mag. Given P1's EW and $\Delta m$ corrected for the trend in the EMD, and allowing for a $5 \sigma$ detection, the corresponding minimum $\Delta m$ and $\mathrm{EW}$ are $0.35 \mathrm{mag}$ and $25 \AA$, respectively. Values of $\Delta m$ corresponding to the scatter of the data ( $0.07 \mathrm{mag}$ ) result in EWs of under $10 \AA$, implying that weak-lined T Tauri stars could not be detected with data of this precision. It would be instructive to produce EMDs containing known CTTSs with emission lines of varying strengths in their spectra, to investigate more robustly the minimum continuum $\mathrm{Br} \gamma$ emission required for a positive detection and how precise the data need to be.

\subsection{CO Emission}

Spectra at various locations across the association map revealed a dip between two peaks that shifted between -15 and $-16.5 \mathrm{~km} \mathrm{~s}^{-1}$. The ambient cloud velocity is in this range, in good agreement with what Yang \& Fukui (1992) and Leisawitz et al. (1989) found in their surveys that mapped ${ }^{12} \mathrm{CO},{ }^{13} \mathrm{CO}$, and $\mathrm{C}^{18} \mathrm{O}$ emission (all $J=1-0$ ). The association map also showed a weak blueshifted outflow that may be related to object A7, the Class I source nearest the peak CO emission. The morphology of the outflow is unlike those in the Perseus molecular cloud, for example, studied by Curtis et al. (2010), and in W5, studied by Ginsburg et al. (2011), who investigated several cometary clouds and pillars using HARP and found dozens of outflow candidates, most of which had low masses. While the slightly elongated shape is similar to some outflow lobes in W5, only the blueshifted lobe is detected. Its strength and morphology indicate that it is more likely associated with A7 than the nearby suspected SCUBA-2 Class 0 source, and its orientation is toward the observer. There may be a redshifted outflow lobe, but it is hidden, according to this scenario. A core of emission corresponding to a velocity of $-17 \mathrm{~km} \mathrm{~s}^{-1}$ is apparent and is within 7 arcsec of the submillimeter emission that may be associated with A7. This velocity is somewhat larger than the systemic velocity of $-16.4 \mathrm{~km} \mathrm{~s}^{-1}$, but not unreasonably so given the width of the line (see Figure 6) and extent of the self-absorption across the region.

Does the outflow scenario make sense with what was obtained for the SED fit? The viewing angle and opening cavity angle of the best-fitting model were $49^{\circ}$ and $18^{\circ}$, respectively. A situation may be visualized wherein the material is being funneled through the cavity, and given the orientation, the material would be transported somewhat toward the viewer (which also would account for the slightly elongated contours in Figure 2).

The optical and mid-IR images of the pillar show structure that is reminiscent of that seen in similar pillars in the W5 region, 
for example; however, our analysis indicates that no outflows are apparent, which is consistent with the evolutionary class of P1. There is a case for multiple small pillars or turbulent structure, as opposed to rotation, given the appearance of the velocity map in Figure 7, the velocity dispersion map, and the morphology of the pillar at optical wavelengths. We believe that the pillar is being affected more by its surroundings than by $\mathrm{P} 1$.

In their study described in the Introduction, Yang \& Fukui (1992) mentioned the presence of emission features in their maps ranging from 0 to $-8 \mathrm{~km} \mathrm{~s}^{-1}$ that are ubiquitous over their survey. They concluded that the features originate in nearby molecular clouds, and there was no evidence in their data of a physical connection between the $-5 \mathrm{~km} \mathrm{~s}^{-1}$ component and the cloud of interest. This component is observed in both regions studied here, but it does not present as a component of the pillar itself, which indicates that the pillar is in the foreground.

\subsection{YSO Distribution and Triggered Star Formation}

The leftmost image of Figure 1 shows a small, partially cleared cavity surrounding Berkeley 59, itself residing near to the southern edge of a larger shell encompassing most of the image. The larger shell was termed the Cep Loop by Rossano et al. (1983), who studied it in $\mathrm{H}_{2} \mathrm{CO}$ and proposed an evolutionary scenario for the region: the formation of several $\mathrm{OB}$ stars (now dispersed) first occurred near the center of what now is the Cep Loop, and the subsequent pressure on the remaining molecular cloud formed the shell and triggered the formation of Berkeley 59 from the fragments of the cloud southeast of the OB stars. Berkeley 59 in turn may be responsible for triggering a third generation of stars.

The ability to confirm the triggering mechanism involved in secondary star formation requires knowledge of the YSO distribution and a timescale for the history of the region in question. Conclusions by Koenig et al. (2012), as well as those by Elmegreen (2000, who studied star formation in the Large Magellanic Cloud) and Barentsen et al. (2011, who used their IPHAS survey data of IC 1396 to establish an age gradient as a function of distance from the triggering source), indicate that the triggering process is a rapid one. In general, age differences between the first and second generation of stars average $2 \mathrm{Myr}$, for shell expansion distances of $5 \mathrm{pc}$. The pillar is located approximately $4 \mathrm{pc}$ from Berkeley 59 as seen in projection and, at an age of $\sim 0.8 \mathrm{Myr}$ as derived from the SED fits, is about 1.2 Myr younger than Berkeley 59. The YSOs in the association are approximately 3 pc from Berkeley 59 as seen in projection and have ages ranging from 0.001 and $0.08 \mathrm{Myr}$ for A10 and A7, respectively, to over 4 Myr for A2 and A6. In light of this, it is possible that Berkeley 59 is the trigger for the formation of P1, but it is less clear whether it is the trigger for the association. If the four association YSOs do in fact form a small cluster, and assuming that the ages are approximately correct, then Berkeley 59 is unlikely to have been the trigger for their formation, since A2 and A6 are too old. Another alternative is that A2 and A6 are simply background sources given their distances and extinctions, in which case it is possible that Berkeley 59 initiated the formation of A7 and A10.

Koenig et al. (2012) studied several clouds and pillars in the Cep Loop, as well as in other regions, and found that the number and distribution of YSOs appeared to scale with the mass (or physical size) of the star-forming region: larger regions were able to support the formation of more YSOs, while the smallest hosted one or at most a few sources. This conclusion applies to our results as well. The pillar in which $\mathrm{P} 1$ resides is comparable in physical size to others in the Cep Loop that contain a single source (cloud mass and density play important roles in the number and masses of stars formed, but without this information, a size comparison must suffice). Furthermore, the darkest part of the cloud (as seen on optical images) in which the association resides is comparable in size $(\sim 1 \mathrm{pc})$ to other regions studied by Koenig et al. (2012) that also contain small groups of only a few YSOs each. As mentioned in the Introduction, the RDI process is one of the main mechanisms for triggered star formation being discussed in the literature. Those authors found that the RDI process was best supported by their data, given the derived surface density distribution of YSOs. There is also support for this scenario from the morphology of the region: several small pillars (including the one hosting P1) point directly toward Berkeley 59, into the lower-density cavity that appears to have been carved out by the pressure of the cluster's massive stars. Since the association is deeply embedded in a cloud, it is unclear which process is responsible in that case. The cloud appears to be undergoing a compression from both Berkeley 59 and the remnants of the original OB stars believed to have existed at the center of the Cep Loop. Thus, it is possible that the original massive stars, and not Berkeley 59, are responsible for triggering the formation of the YSOs in the cloud, but the triggering mechanism is uncertain.

\section{CONCLUSIONS}

We have identified a small association of YSOs in a dark cloud northwest of the young open cluster Berkeley 59 and another YSO at the head of a pillar located south of the cluster. A $J-H$, $H-K_{s}$ diagram reveals that all the sources exhibit IR excesses. Four sources in the association and the pillar YSO appear in most of the WISE passbands. 2MASS, WISE, and SCUBA-2 continuum data indicate that two of the association objects are of evolutionary Class I, while two exhibit flat/Class II spectra, which is confirmed by model fits to the sources' SEDs: the least-evolved sources possess substantial disks and accreting envelopes, while the more evolved sources have considerably smaller disk masses and in some cases no envelope. The leastevolved objects were classified under the assumption that the SCUBA-2 sources identified were directly associated with the IR sources. In one case, it is possible that the SCUBA-2 source is unrelated to the IR source and is in fact a consequence of a more deeply embedded Class 0 object. An additional suspected Class 0 object is nearby, in the same cloud. The YSO in the pillar is classified as flat/Class II. An EMD using narrowband IR and $\mathrm{H} \alpha$ IPHAS data suggest that it is a CTTS candidate.

The ${ }^{12} \mathrm{CO}(J=3-2)$ emission shows that both regions are physically associated with Berkeley 59 on the basis of their velocities: the systemic velocities of the regions are approximately $-16 \mathrm{~km} \mathrm{~s}^{-1}$, which corresponds to the velocity of the cluster, at $-15.7 \mathrm{~km} \mathrm{~s}^{-1}$. Outflows are only present in the association region, wherein a weak blueshifted outflow was detected. At this point it is unclear whether the outflow is associated with object A7 or the possible embedded Class 0 source. A strong core at the velocity of $-17 \mathrm{~km} \mathrm{~s}^{-1}$ is within 7 arcsec of the submillimeter emission. The pillar exhibits structure that is supported by a variation in velocity across its face: the velocity field map shows two regions at a slightly different velocity $\left(-15 \mathrm{~km} \mathrm{~s}^{-1}\right)$ than the head of the pillar $\left(-16 \mathrm{~km} \mathrm{~s}^{-1}\right)$, and the velocity dispersion is substantial only in these two regions.

It is likely that P1 was formed in a round of secondary star formation instigated by Berkeley 59, according to the RDI 
process. The situation is unclear for the association objects. We speculate that the cloud in which they reside is being compressed by Berkeley 59 to the east and by a since-dispersed OB association to the west that may have been responsible for the formation of the Cep Loop and Berkeley 59 itself. Given this and the spread in ages for the association YSOs $(0.008$ to $>4 \mathrm{Myr}$ ), it is not possible to determine whether the original OB stars or Berkeley 59 triggered the formation of those YSOs, or which triggering mechanism was responsible.

This publication makes use of data products from the Two Micron All-Sky Survey, which is a joint project of the University of Massachusetts and the Infrared Processing and Analysis Center/California Institute of Technology, funded by the National Aeronautics and Space Administration and the National Science Foundation, and data products from the Wide-field Infrared Survey Explorer, which is a joint project of the University of California, Los Angeles, and the Jet Propulsion Laboratory/ California Institute of Technology, funded by the National Aeronautics and Space Administration.

The Observatoire du Mont Mégantic is funded by the Université de Montréal, Université Laval, the Natural Sciences and Engineering Research Council of Canada (NSERC), the Fond québécois de la recherche sur la Nature et les technologies (FQRNT), and the Canada Economic Development program.

The James Clerk Maxwell Telescope is operated by the Joint Astronomy Center (JAC) on behalf of the Science and Technology Facilities Council of the United Kingdom, the Netherlands Organisation for Scientific Research, and the National Research Council of Canada. Additional funds for the construction of SCUBA-2 were provided by the Canada Foundation for Innovation.

We thank É. Artigau for advice with the OMM data and are grateful to I. Coulson, T. Jenness, H. Parsons, J. Dempsey, R. Redman, and J. di Francesco for their advice in writing the JCMT observing proposals and their assistance with the data reduction and analysis.

We thank the anonymous referee for a careful reading of this paper and suggestions that improved the scope of the paper and its conclusions.

\section{REFERENCES}

Allen, L. E., Calvet, N., d'Alessio, P., et al. 2004, ApJS, 154, 363

Artigau, É., Lamontagne, R., Doyon, R., \& Malo, L. 2010, Proc. SPIE, 7737,41

Barentsen, G., Vink, J. S., Drew, J. E., et al. 2011, MNRAS, 415, 103

Beck, T. L., Bary, J. S., \& McGregor, P. J. 2010, ApJ, 722, 1360

Buckle, J. V., Hills, R. E., Smith, H., et al. 2009, MNRAS, 399, 1026

Cavanagh, B., Jenness, T., Economou, F., \& Currie, M. J. 2008, AN, 329, 295

Chapin, E. L., Berry, D. S., Gibb, A. G., et al. 2013, MNRAS, 430, 2545

Corradi, R. L. M., Rodríguez-Flores, E. R., Mampaso, A., et al. 2008, A\&A, 480, 409

Curtis, E. I., Richer, J. S., Swift, J. J., \& Williams, J. P. 2010, MNRAS, 408, 1516
Dempsey, J. T., Friberg, P., Jenness, T., et al. 2013, MNRAS, 430, 2534

Drabek, E., Hatchell, J., Friberg, P., et al. 2012, MNRAS, 426, 23

Drew, J. E., Greimel, R., Irwin, M. J., et al. 2005, MNRAS, 362, 753

Egan, M. P., Price, S. D., \& Kraemer, K. E. 2003, BAAS, 203, 5708

Elmegreen, B. G. 2000, ApJ, 530, 277

Elmegreen, B. G. 2009, in IAU Symp. 254, The Galaxy Disc in Cosmological Context, ed. J. Andersen, J. Bland-Hawthorn, \& B. Nordström (Cambridge: Cambridge Univ. Press), 289

Elmegreen, B. G. 2011, in EAS Publ. Ser. 51, Star Formation in the Local Universe, ed. C. Charbonnel \& T. Montmerle (Cambridge: Cambridge Univ. Press), 45

Elmegreen, B. G., \& Lada, C. J. 1977, ApJ, 214, 725

Gahm, G. F., Carlqvist, P., Johansson, L. E. B., \& Nikolić, S. 2006, A\&A, 454, 201

Ginsburg, A., Bally, J., \& Williams, J. P. 2011, MNRAS, 418, 2121

Greene, T. P., Wilking, B. A., André, P., Young, E. T., \& Lada, C. J. 1994, ApJ, 434, 614

Gutermuth, R. A., Megeath, S. T., Myers, P. C., et al. 2009, ApJS, 184, 18

Holland, W. S., Bintley, D., Chapin, E. L., et al. 2013, MNRAS, 430, 2513

Ishihara, D., Onaka, T., Kataza, H., et al. 2010, A\&A, 514, A1

Kataza, H., Alfageme, C., Cassatella, A., et al. 2010, AKARI-IRC Point Source Catalogue Release Note Ver. 1.0 (Japan), http://www.ir.isas.jaxa.jp/AKARI/ Observation/PSC/Public/RN/AKARI-IRC_PSC_V1_RN.pdf

Koenig, X. P., Leisawitz, D. T., Benford, D. J., et al. 2012, ApJ, 744, 130

Kun, M., Kiss, Z. T., \& Balog, Z. 2008, in Handbook of Star Forming Regions, Vol. 1, ed. B. Reipurth (San Francisco, CA: ASP), 136

Lada, C. J. 1987, in IAU Symp. 115, Star Forming Regions, ed. M. Peimbert \& J. Jugaku (Dordrecht: Reidel), 1

Leisawitz, D., Bash, F. N., \& Thaddeus, P. 1989, ApJS, 70, 731

Liu, T., Janes, K. A., \& Bania, T. M. 1989, AJ, 98, 626

MacConnell, D. J. 1968, ApJS, 16, 275

Majaess, D. J., Turner, D. G., Lane, D. J., \& Moncrieff, K. E. 2008, JAVSO, 36, 90

Meyer, M. R., Calvet, N., \& Hillenbrand, L. A. 1997, AJ, 114, 288

Muzerolle, J., Hartmann, L., \& Calvet, N. 1998, AJ, 116, 2965

Najita, J., Carr, J. S., \& Tokunaga, A. T. 1996, ApJ, 456, 292

Osterbrock, D. E., \& Ferland, G. J. 2006, in Astrophysics of Gaseous Nebulae and Active Galactic Nuclei, ed. J. Murdzek (2nd ed.; Sausalito, CA: University Science Books), 78

Pandey, A. K., Sharma, S., Ogura, K., et al. 2008, MNRAS, 383, 1241

Reipurth, B. 1983, A\&A, 117, 183

Robitaille, T. P., Meade, M. R., Babler, B. L., et al. 2008, AJ, 136, 2413

Robitaille, T. P., Whitney, B. A., Indebetouw, R., \& Wood, K. 2007, ApJS, 169,328

Robitaille, T. P., Whitney, B. A., Indebetouw, R., Wood, K., \& Denzmore, P. 2006, ApJS, 167, 256

Rossano, G. S., Grayzeck, E. J., \& Angerhofen, P. E. 1983, AJ, 88, 1835

Shara, M. M., Moffat, A. F. J., Gerke, J., et al. 2009, AJ, 138, 402

Skrutskie, M. F., Cutri, R. M., Stiening, R., et al. 2006, AJ, 131, 1163

Stetson, P. B. 1987, PASP, 99, 191

Straižys, V., \& Laugalys, V. 2007, BaltA, 16, 327

Straižys, V., \& Laugalys, V. 2008, BaltA, 17, 1

Straižys, V., \& Laugalys, V. 2009, BaltA, 18, 19

Straižys, V., \& Lazauskaitè, R. 2008, BaltA, 17, 277

Strom, K. M., Newton, G., Strom, S. E., et al. 1989, ApJS, 71, 183

Varricatt, W. P., Davis, C. J., Ramsay, S., \& Todd, S. P. 2010, MNRAS, 404, 661

Whitney, B. A., Wood, K., Bjorkman, J. E., \& Cohen, M. 2003, ApJ, 598, 1079

Wright, E. L., Eisenhardt, P. R. M., Mainzer, A. K., et al. 2010, AJ, 140, 1868

Yang, J., \& Fukui, Y. 1992, ApJ, 386, 618

Yang, J., Fukui, Y., Umemoto, T., Ogawa, H., \& Chen, H. 1990, ApJ, 362,538

Zavagno, A., Deharveng, L., Comerón, F., et al. 2006, A\&A, 446, 171 